\title{
On the Douady Space of a Compact Complex Space in the Category $\mathscr{C}$, II
}

By

\author{
Akira FUJIKI*
}

\section{Introduction}

This is a continuation of our previous paper [4]. Let $f: X \rightarrow S$ be a proper morphism of complex spaces and $\mathscr{E}$ a coherent analytic sheaf on $X$. Then we denote by $D_{X / S}(\mathscr{E})$ the Douady space of flat quotients of $\mathscr{E}$ over $S ; D_{X / S}(\mathscr{E})$ represents the functor $\boldsymbol{D}_{X / S}(\mathscr{E})$ : $(\mathrm{An} / S)^{\circ} \rightarrow$ Sets defined by $\boldsymbol{D}_{X / S}(\mathscr{E})(T):=$ the set of coherent quotients $\mathscr{F}$ of $\mathscr{E}_{T}$ (the pull-back of $\mathscr{E}$ to $X \times{ }_{S} T$ ) such that $\mathscr{F}$ is flat over $T$.

We say that $f$ is a $\mathscr{C}$-morphism ( $f$ belongs to $\mathscr{C} / S$ in the terminology of [4]) (resp. is Moishezon) if $X_{\text {red }}$ is a meromorphic image over $S$ of a complex space $\tilde{X}$ which is proper and locally Kähler (resp. locally projective) over $S$ (cf. [4, (2.1) (resp. (1.2))]). Then the purpose of the present paper is to prove the following theorem which generalizes Theorem in [4]:

Theorem. Let $f: X \rightarrow S$ be a proper morphism of complex spaces and $\mathscr{E}$ a coherent analytic sheaf on $X$. Let $b: D_{X / S}(\mathscr{E})_{\mathrm{red}} \rightarrow S$ be the natural morphism. Suppose that $f$ is a $\mathscr{C}$-morphism (resp. is Moishezon). Then for any relatively compact subdomain $Q$ of $S$ and for any irreducible component $A$ of $b^{-1}(Q)$ the induced morphism $\left.b\right|_{A}: A \rightarrow Q$ is proper and is a $\mathscr{C}$-morphism (resp. is Moishezon).

Corollary. Let $X$ be a compact complex space in $\mathscr{C}$. Then any irreducible component $D_{\alpha}$ of the reduced Douady space $D_{X, \text { red }}$ is again compact and belongs to $\mathscr{C}$. If, further, $X$ is Moishezon, $D_{\alpha}$ is again Moishezon.

Communicated by S. Nakano, February 10, 1983.

* Department of Mathematics, Yoshida College, Kyoto University, Kyoto 606, Japan. 
As is mentioned above, Theorem was shown in [4] in the case where $\mathscr{E}=\mathcal{O}_{X}$ (so that $D_{X / S}(\mathscr{E})$ reduces to the ordinary relative Douady space $D_{X / S}$ of $X$ over $S$ ) and the universal subspace $Z_{X / S} \subseteq$ $X \times{ }_{S} D_{X / S}$ restriced over $A \subseteq D_{X / S}$ is reduced. On the other hand, the properness of $\left.b\right|_{A}$ in general was shown in [3, Theorem 5.2] under the assumption that $f$ is a Kähler morphism, and more generally it was asserted in [3] with a sketch of proof in the case where $f$ is a $\mathscr{C}$-morphism. (See however Remark 2 at the end of this paper.) Indeed, Theorem above follows readily from the result of [4] above if we re-examine the proof of Theorem 5.2 in [3] (the part where the general case is reduced to the case where $\mathscr{E}=$ $\mathcal{O}_{X}$ and $Z_{X / S}$ is reduced over $A$ ), except that the proof there contains a gap in the formulation and proof of Lemma 5.8. The main point of our proof is thus nothing but to fill that gap in a way suitable for the proof of the above theorem.

In Section 1 we first construct the universal space of extensions of a given coherent analytic sheaf by another coherent analytic sheaf satisfying some additional conditions, and then construct the natural compactification of it. Then in Section 2 using the results obtained in Section 1 we prove a revised form of [3, Lemma 5.8] (cf. Lemma 12 below) and then prove Theorem above, essentially following the proof of [3, Theorem 5.2]. Also some other corrections to [3] will be given (cf. a remark after Lemma 14 and Remark 2). We follow mainly the notations and terminologies of [4]. Also we recall the following notation from [3]. Let $f: X \rightarrow S$ be a morphism of complex spaces and $\mathscr{E}$ a coherent analytic sheaf on $X$. Let $\nu: T \rightarrow S$ be a morphism of complex spaces. Then the pullback of $\mathscr{E}$ to $X_{T}:=X \times{ }_{S} T$ will usually be denoted by $\mathscr{E}_{T}$ (with $\nu$ understood). Further $(\mathrm{An} / S)$ denotes the category of complex spaces over $S$. A reduced and irreducible complex space is called a complex variety. A Zariski open subset is in general assumed to be nonempty unless otherwise is explicitly stated. The following result of Frisch [2] will be used without reference throughout the paper; for any $f$ : $X \rightarrow S$ and $\mathscr{E}$ as above with $S$ reduced and with $f$ proper there exists a dense Zariski open subset $U$ of $S$ over which $\mathscr{E}$ is $f$-flat. 


\section{$\S 1$. Space of Completions of a Diagram}

1.1. In this subsection we gather some preliminary results.

a) Let $T$ be a complex space and $\mathscr{F}$ a coherent analytic sheaf on $T$. Let $U \subseteq T$ be a Zariski open subset. Then a holomorphic section $s \in H^{0}(U, \mathscr{F})$ of $\mathscr{F}$ over $U$ is said to be extended meromorphically to $T$ if there exists a coherent sheaf of ideals $\mathscr{I}$ of $\mathcal{O}_{T}$ such that $\operatorname{supp} \mathcal{O}_{T} / \mathscr{I} \subseteq A:=T-U$ and that for any open subset $V$ of $T$ and any element $b \in H^{0}(V, \mathscr{I}), b_{0} s_{0}$ extends to a holomorphic section of $\mathscr{F}$ over the whole $V$, where supp denotes the support and the subscript 0 denotes the restriction to $U \cap V$. By the Hibert Nullstellensatz we get

Lemma 1. Suppose that there exists an effective Cartier divisor $D$ on $T$ whose support coincides with $A$. Then $s$ is extended meromorphically to $T$ if and only if for any relatively compact subdomain $W \subseteq T$ there exist an integer $n>0$ and an element $s^{*} \in H^{0}(W, \mathscr{F}(n D))$ which is an extension of $\left.s\right|_{U \cap W}$ with respect to the natural isomorphism $\mathscr{F}(n D) \cong \mathscr{F}$ on $U$ where $\mathscr{F}(n D)=\mathscr{F} \otimes \mathcal{O}\left([D]^{n}\right)$ with $[D]$ the holomorphic line bundle defined by $D$.

Let $T$ be a complex space. Let $U \subseteq T$ be a Zariski open subset. Let $\mathscr{F}, \mathscr{G}$ be coherent analytic sheaves on $T$. Then we say that $\mathscr{F}$ and $\mathscr{G}$ are meromorphically equivalent and isomorphic on $U$ if there exist coherent analytic sheaves $\mathscr{F}_{0}, \ldots, \mathscr{F}_{m}$ on $T$ such that 1) $\mathscr{F}_{0}=\mathscr{F}$, $\mathscr{F}_{m}=\mathscr{G}$ and 2) for each $1 \leqq i \leqq m$, there exists a homomorphism $\mathscr{F}_{i-1}$ $\rightarrow \mathscr{F}_{i}$ or $\mathscr{F}_{i} \rightarrow \mathscr{F}_{i-1}$ which is isomorphic over $U$.

Lemma 2. Suppose that $\mathscr{F}$ and $\mathscr{G}$ are meromorphically equivalent and isomorphic on $U$. Let $s \in H^{0}(U, \mathscr{F})$. Let $s^{\prime} \in H^{0}(U, \mathscr{G})$ be the section corresponding to $s$ via the natural isomorphism $\mathscr{F} \cong \mathscr{G}$ on $U$. Then $s$ is extended meromorphically to $T$ if and only if so is $s^{\prime}$.

Proof. We can reduce the proof immediately to the case where there exists a homomorphism $u: \mathscr{F} \rightarrow \mathscr{G}$ which is isomorphic over $U$. Then the assertion for $s$ clearly implies that for $s^{\prime}$. So suppose that $s^{\prime}$ is extended meromorphically to $T$. Let $\mathscr{I}^{\prime}$ be the coherent sheaf of 
ideals of $T$ as in the above definition of meromorphic extension for $\left(\mathscr{G}, s^{\prime}\right)$. Let $\mathscr{C}$ be the cokernel of $u$. Then the support of $\mathscr{C}$ is contained in $A$. Hence if $\mathscr{J}$ is the ideal sheaf of annihilators of $\mathscr{C}$, then $\operatorname{supp} \mathcal{O}_{T} / \mathscr{J} \cong A$. We set $\mathscr{I}=\mathscr{I}^{\prime} \mathscr{J}$. We then show that for any open subset $V \leqq T$ and any $b \in H^{0}(V, \mathscr{I}) b_{0} s_{0}$ extends to a holomorphic section of $\mathscr{F}$ over $V$. Since the problem is local we may assume that $V$ is Stein and $b$ is of the form $b=b_{1} b_{2}$, where $b_{1} \in$ $H^{0}\left(V, \mathscr{I}^{\prime}\right)$ and $b_{2} \in H^{0}(V, \mathscr{J})$. First, $b_{10} s_{0}^{\prime}$ extends to a holomorphic section, say $w$, of $\mathscr{G}$ over $V$. Then $b_{2} w \in H^{0}(V, \operatorname{Im} u)$ where $\operatorname{Im}$ denotes the image. Hence there exists an element $t \in H^{0}(V, \mathscr{F})$ which is mapped to $b_{2} w$. Then since $u$ is isomorphic on $U, t_{0}=b_{0} s_{0}$. q. e.d.

b) (cf. [6]). Let $S$ be a complex space and $\mathscr{E}$ a coherent analytic sheaf on $S$. Then the linear fiber space $L(\mathscr{E})$ over $S$ associated to $\mathscr{E}$ is a complex space over $S$ which represents the functor $F$ : $(\mathrm{An} / S)^{\circ} \rightarrow($ Vector Spaces $/ \boldsymbol{C})$ defined by

$$
F(T):=\operatorname{Hom}_{\mathscr{O}_{T}}\left(\nu^{*} \mathscr{E}, \mathcal{O}_{T}\right)=H^{0}\left(T,\left(\nu^{*} \mathscr{E}\right)^{*}\right)
$$

where $\nu: T \rightarrow S$ is any morphism and * denotes taking the dual. From the definition we get

$$
\mathscr{S}(L(\mathscr{E})) \cong \mathscr{E}^{*}
$$

where in general for a linear fiber space $L$ over $S, \mathscr{S}(L)$ denotes the sheaf of germs of holomorphic sections of $L$. When $\mathscr{E}$ is locally free, we see that $L\left(\mathscr{E}^{*}\right)$ is a vector bundle over $S$ and it represents the functor $V:(\mathrm{An} / S)^{\circ} \rightarrow$ Sets defined by

$$
V(T):=H^{0}\left(T, \nu^{*} \mathscr{E}\right)
$$

with $\nu$ as above. In this case we call $L\left(\mathscr{E}^{*}\right)$ the vector bundle associated to the locally free sheaf $\mathscr{E}$ (against the terminology of [6]).

Further recall that a projective fiber space $\mathbb{P}(\mathscr{E})$ over $S$ associated to $\mathscr{E}$ is the complex space over $S$ which represents the functor $P$ : $(\mathrm{An} / S)^{\circ} \rightarrow$ Sets defined by

$$
P(T):=\text { the set of invertible quotients } \mathscr{L} \text { of } \nu^{*} \mathscr{E} .
$$

Lemma 3. Let $S$ be a complex space and $\mathscr{E}$ a coherent analytic sheaf on $S$. Let $\pi: L=L(\mathscr{E}) \rightarrow S$ be the natural projection and $\alpha \in H^{0}(L$, 
$\left.\left(\pi^{*} \mathscr{E}\right)^{*}\right)$ the universal section. Let $\bar{\pi}: \mathbb{P}=\mathbb{P}\left(\mathscr{E} \oplus \mathcal{O}_{S}\right) \rightarrow S$ be the projective fiber space associated to $\mathscr{E} \oplus \mathcal{O}_{s}$. Then there exists a natural Zariski open inclusion $L \subseteq \mathbb{P}$ over $S$ such that $\alpha$ extends meromorphically to $\boldsymbol{P}$ (as a section of $\left.\left(\bar{\pi}^{*} \mathscr{E}\right)^{*}\right)$.

Proof. Let $\beta: \bar{\pi}^{*}\left(\mathscr{E} \oplus \mathcal{O}_{S}\right) \rightarrow \mathscr{L}$ be the universal quotient on $\mathbb{P}$. Let $j: L \rightarrow \mathbb{P}$ be the $S$-morphism induced by the quotient homomorphism $(\alpha+i d): \pi^{*} \mathscr{E} \oplus \mathcal{O}_{P}\left(\cong \pi^{*}\left(\mathscr{E} \oplus \mathcal{O}_{P}\right)\right) \rightarrow \mathcal{O}_{P}$ on $L$ together with the universality of $\boldsymbol{P}$. On the other hand, let $s \in H^{0}(\boldsymbol{P}, \mathscr{L})$ be the image by $\beta$ of the constant section $(0,1) \in H^{0}\left(\mathbb{P}, \bar{\pi}^{*} \mathscr{E} \oplus \mathcal{O}_{\boldsymbol{P}}\right)=$ $H^{0}\left(\mathbb{P}, \bar{\pi}^{*} \mathscr{E} \oplus \mathcal{O}_{s}\right)$. Let $D$ be the divisor of zero of $s$ and $W=\boldsymbol{P}-D$. Then on $W, s$ defines the canonical isomorphism $\phi_{s}: \mathcal{O}_{W} \cong \mathscr{L}$ $\left(\cong \mathcal{O}_{P}(D)\right)$. Then $\left.\phi_{s}^{-1}\left(\left.\beta\right|_{\bar{\pi}^{+} \mathscr{E}}\right)\right|_{W}: \bar{\pi}^{*} \mathscr{E} \rightarrow \mathcal{O}_{W}$ defines an $S$-morphism $\mu: W \rightarrow L$ by the universality of $L$. It is then easy to see that $j(L) \subseteq W$ and that $j$ and $\mu$ are inverse to each other. Now let $\bar{\alpha}: \bar{\pi}^{*} \mathscr{E} \rightarrow \mathcal{O}_{P}(D)$ be the composite of $\left.\beta\right|_{\pi^{*} \mathscr{E}}$ and the isomorphism $\mathscr{L} \cong \mathcal{O}_{P}(D)$. Then $\bar{\alpha} \in \operatorname{Hom}_{\mathcal{O}_{P}}\left(\bar{\pi}^{*} \mathscr{E}, \mathcal{O}_{P}(D)\right) \cong \operatorname{Hom}_{\mathcal{O}_{P}}\left(\bar{\pi}^{*} \mathscr{E}, \mathcal{O}_{P}\right)(D)$ is considered as a holomorphic extension of $\alpha$ to $\mathbb{P}$. Then the lemma follows from Lemma 1.

q. e. d.

c) Let $f: X \rightarrow S$ be a morphism of complex spaces. Let $\mathscr{F}_{1}$ and $\mathscr{F}_{2}$ be coherent analytic sheaves on $X$. Let $x$ be any point of $X$. Consider an exact sequence

$$
\mathcal{O}_{X}^{\oplus q} \stackrel{u}{\longrightarrow} \mathcal{O}_{X}^{\oplus p} \stackrel{\rho}{\longrightarrow} \mathscr{F}_{1} \longrightarrow 0
$$

defined in a neighborhood of $x_{0}$. Then applying $\mathscr{H}_{\mathrm{C}_{\mathrm{m}} \boldsymbol{O}_{X}}\left(, \mathscr{F}_{2}\right)$ to (*) we get a homomorphism

$$
v: \mathscr{F}_{2}^{\oplus p} \longrightarrow \mathscr{F}_{2}^{\oplus q} \text {. }
$$

Then we say that a pair $\left(\mathscr{F}_{1}, \mathscr{F}_{2}\right)$ satisfies the condition (C) at $x$ if both $\operatorname{Im} v$ and Coker $v$ are $f$-flat at $x$ for some exact sequence (*) as above. We say that $\left(\mathscr{F}_{1}, \mathscr{F}_{2}\right)$ satisfies (C) over an open subset $U$ of $S$ if $\left(\mathscr{F}_{1}, \mathscr{F}_{2}\right)$ satisfies $(\mathrm{C})$ at any point of $X_{U}$.

Lemma 4. Suppose that $\mathscr{F}_{2}$ is $f$-flat at $x$. Then the condition that $\operatorname{Im} v$ and Coker $v$ are $f$-flat at $x$ is independent of the exact sequence (*) above. 
Proof. Let $r, s$ be nonnegative integers with $r \leqq s$. Let $\pi: \mathcal{O}_{\bar{X}}^{\oplus s} \rightarrow$ $\mathcal{O} \underset{X}{\oplus r}$ be the projection to the first $r$ factors. Let $u^{\prime}=u \oplus \pi$ and $\rho^{\prime}=\rho p_{1}$, where $u$ and $\rho$ are as in (*) and $p_{1}: \mathcal{O} \frac{\oplus(r+p)}{X} \rightarrow \mathcal{O} \oplus_{X}^{\oplus p}$ is the projection. Then the resulting exact sequence

$$
\mathcal{O}_{X}^{\oplus(s+q)} \stackrel{u^{\prime}}{\longrightarrow} \mathcal{O} \underset{X}{\oplus(r+p)} \stackrel{\rho^{\prime}}{\longrightarrow} \mathscr{F}_{1} \longrightarrow 0
$$

is called the $(r, s)$-modification of $(*)$. Let $v^{\prime}: \mathscr{F}_{2}^{\oplus(r+p)} \rightarrow \mathscr{F}_{2}^{\oplus(s+q)}$ be the homomorphism defined by $u^{\prime}$. Then it is immediate to see that Ker $v^{\prime} \cong \operatorname{Ker} v$ and Coker $v^{\prime} \cong$ Coker $v \oplus \mathscr{F}_{2}^{\oplus(s-r)}$. Hence under our assumption the condition is independent of passing to any $(r, s)$ modification of $(*)$. Then the lemma follows from the fact that any two exact sequences as $(*)$ are isomorphic (over the identity of $\left.\mathscr{F}_{1}\right)$ at $x$ if we pass to a suitable $(r, s)$-modification of each sequence (cf. the proof of Theorem in [7, p. 102]). q. e.d.

Using this lemma we shall show the following:

Lemma 5. Assume that $f$ is proper. Then there exists a dense Zariski open subset $U$ of $S$ such that $\left(\mathscr{F}_{1}, \mathscr{F}_{2}\right)$ satisfies the condition $(\mathrm{G})$ over $U$.

Proof. Take a locally finite open covering $\mathfrak{W}=\left\{W_{\alpha}\right\}$ of $X$ such that on each $W_{\alpha}$ we get an exact sequence $\mathcal{O}_{X}^{\oplus q_{\alpha}} \stackrel{u_{\alpha}}{\longrightarrow} \mathcal{O}_{X}^{\oplus p_{\alpha}} \longrightarrow \mathscr{F}_{1} \longrightarrow 0$. Let $v_{\alpha}: \mathscr{F}_{2}^{\oplus p_{\alpha}} \longrightarrow \mathscr{F}_{2}^{\oplus q_{\alpha}}$ be the homomorphism associated to $u_{\alpha}$. Let $T_{\alpha}=\left\{x \in W_{\alpha} ; \operatorname{Im} v_{\alpha}\right.$ and Coker $v_{\alpha}$ are not $f$-flat at $\left.x\right)$. Then by Frisch $T_{\alpha}$ is an analytic subset of $W_{\alpha}$ such that $f\left(T_{\alpha}\right)$ is 'negligible' in $S$ (cf. [2, Prop. (IV, 14)]). Let $U_{1}$ be a Zariski open subset of $S$ over which $\mathscr{F}_{2}$ is $f$-flat. Then by Lemma $4 T_{\alpha}=T_{\beta}$ on $W_{\alpha} \cap W_{\beta} \cap$ $X_{U_{1}}$. Hence $T_{\alpha}$ define a global analytic subset $T\left(U_{1}\right)$ on $X_{U_{1}}$. Moreover the closure $T$ of $T\left(U_{1}\right)$ in $X$ is clearly analytic. Let $U_{2}=$ $S-f(T)$. Since $T \subseteq \cup_{\alpha} T_{\alpha}$ and $W$ is locally finite, $f(T)$ is negligible in $S$. Let $U=U_{1} \cap U_{2}$. Then it is clear that $\left(\mathscr{F}_{1}, \mathscr{F}_{2}\right)$ satisfies the condition (C) over $U$, which is Zariski open in $S$. q. e.d.

d) Let $b: X \rightarrow Y$ be a morphism of complex spaces. Let $\mathscr{F}$ and $\mathscr{G}$ be coherent analytic sheaves on $Y$. Then there exists a canonical homomorphism (cf. $[5,0,(6.7 .6)]$ )

$$
b^{\sharp}: b^{*} \mathscr{H}_{o m \mathcal{O}_{Y}}(\mathscr{F}, \mathscr{G}) \longrightarrow \mathscr{H}_{o m \mathcal{O}_{X}}\left(b^{*} \mathscr{F}, b^{*} \mathscr{G}\right) .
$$


In particular when $\mathscr{G}=\mathcal{O}_{Y}$ this reduces to

$$
b^{\sharp}: b^{*} \mathscr{F}^{*} \longrightarrow\left(b^{*} \mathscr{F}\right)^{*} \text {. }
$$

Lemma 6. Let $f: X \rightarrow S$ be a morphism of complex spaces and $\mathscr{F}_{1}, \mathscr{F}_{2}$ coherent analytic sheaves on $X$. Let $\nu: S^{\prime} \rightarrow S$ be a morphism of complex spaces and $\tilde{\nu}: X_{S^{\prime}} \rightarrow X$ the natural projection. Then the canonical homomorphism

$$
\tilde{\nu}^{\#}: \tilde{\nu}^{*} \mathscr{H}_{\mathrm{om} \mathcal{O}_{X}}\left(\mathscr{F}_{1}, \mathscr{F}_{2}\right) \longrightarrow \mathscr{H}_{\mathrm{f} m 0_{X_{S^{\prime}}}}\left(\tilde{\nu}^{*} \mathscr{F}_{1}, \tilde{\nu}^{*} \mathscr{F}_{2}\right)
$$

above is isomorphic if $\left(\mathscr{F}_{1}, \mathscr{F}_{2}\right)$ satisfies the condition (G) over $S$.

Proof. Let $X^{\prime}=X_{S^{\prime}}$. Let $x^{\prime} \in X^{\prime}$ be an arbitrary point. Let $x=$ $\tilde{\nu}\left(x^{\prime}\right)$. Take any exact sequence $(*)$ defined in a neighborhood of $x$ (cf. c)). From this we get the commutative diagram

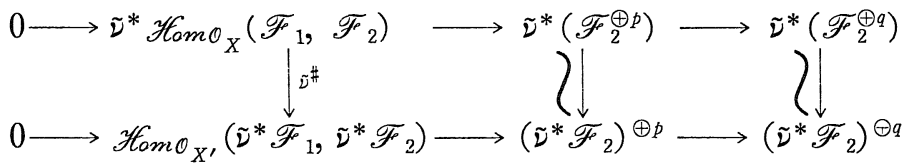

where the top (resp. bottom) sequence is obtained as the pull-back by $\tilde{\nu}$ of the exact sequence

$$
0 \longrightarrow \mathscr{H}_{o m \mathcal{O}_{X}}\left(\mathscr{F}_{1}, \mathscr{F}_{2}\right) \longrightarrow \mathscr{F}_{2}^{\oplus p} \stackrel{v}{\longrightarrow} \mathscr{F}_{2}^{\oplus q}
$$

coming from $(*)$ (resp. by applying $\mathscr{H}_{\operatorname{Com}_{X}}\left(, \tilde{\nu}^{*} \mathscr{F}_{2}\right)$ to the exact sequence $\mathcal{O}_{X^{\prime}}^{\oplus q} \longrightarrow \mathcal{O}_{X^{\prime}}^{\oplus} \longrightarrow \tilde{\mathcal{L}}^{*} \mathscr{F}_{1} \longrightarrow 0$ ). Hence the top (resp. bottom) sequence is exact by virtue of the condition (G) (resp. without any assumption on $\mathscr{F}_{i}$ and $f$ ). The lemma follows from this.

1.2. a) Let $X$ be a complex space. Suppose that we are given a diagram

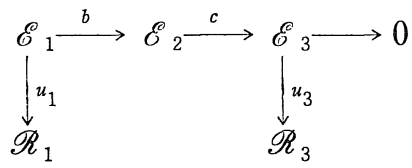

of coherent analytic sheaves on $X$, where the horizontal sequence is exact and $u_{1}, u_{3}$ are surjective. Then we set

$$
\left\{\begin{array}{l}
\mathscr{F}_{i}=\operatorname{Ker} u_{i}, \quad i=1,3 \\
\mathscr{F}_{4}=c^{-1}\left(\mathscr{F}_{3}\right) / b\left(\mathscr{F}_{1}\right) .
\end{array}\right.
$$


Let $\mathscr{K}$ be the kernel of $c$. Then we get an exact sequence

$$
0 \longrightarrow \mathscr{K} \longrightarrow c^{-1}\left(\mathscr{F}_{3}\right) \stackrel{c}{\longrightarrow} \mathscr{F}_{3} \longrightarrow 0 \text {. }
$$

Conversely, $c^{-1}\left(\mathscr{F}_{3}\right)$ is characterized as a submodule of $\mathscr{E}_{2}$ by (6). Further $c$ induces the natural homomorphism $\bar{c}: \mathscr{F}_{4} \longrightarrow \mathscr{F}_{3}$, and $\bar{c}$ in turn induces a homomorphism

$$
\alpha: \mathscr{H}_{\operatorname{tam} \mathbb{C}_{X}}\left(\mathscr{F}_{3}, \mathscr{F}_{4}\right) \longrightarrow \mathscr{H}_{\text {m } \mathcal{O}_{X}}\left(\mathscr{F}_{3}, \mathscr{F}_{3}\right) .
$$

Definition. A completion of (4) is a commutative diagram

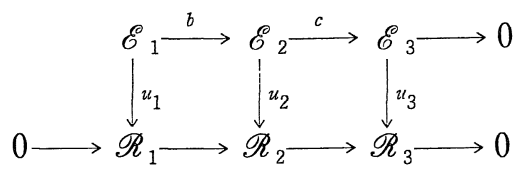

of exact sequences of coherent analytic sheaves on $X$, where $u_{2}$ is surjective as well as $u_{1}, u_{3}$. We define the isomorphisms of two completions in an obvious way; then the set of isomorphism classes is naturally identified with the set of quotients of $\mathscr{E}_{2}$ which fit into the commutative diagram (8).

Lemma 7. Let id $\in \operatorname{Hom}_{\mathcal{O}_{X}}\left(\mathscr{F}_{3}, \mathscr{F}_{3}\right)$ be the identity of $\mathscr{F}_{3}$. Let $M=\alpha^{-1}(i d)$, which is an affine linear subspace of the vector space Hom $_{\mathcal{O}_{X}}\left(\mathscr{F}_{3}, \mathscr{F}_{4}\right)$. Then the set of isomorphism classes of completions of (4) is in natural bijective correspondence with the set $M$.

Proof. Suppose that we are given a completion (8) of (4). Let $\mathscr{F}_{2}$ be the kernel of $u_{2}$. Then $c$ induces an isomorphism $c_{0}: \mathscr{F}_{2} / b\left(\mathscr{F}_{1}\right) \widetilde{\neg}$ $\mathscr{F}_{3}$. Let $j: \mathscr{F}_{2} / b\left(\mathscr{F}_{1}\right) \rightarrow \mathscr{F}_{4}=c^{-1}\left(\mathscr{F}_{3}\right) / b\left(\mathscr{F}_{1}\right)$ be the natural inclusion. Then $j c_{0}^{-1}: \mathscr{F}_{3} \rightarrow \mathscr{F}_{4}$ clearly determines a point of $M$ which depends only on the isomorphism class of (8).

Conversely, given a point $m \in M \subseteq \operatorname{Hom}_{\mathcal{O}_{X}}\left(\mathscr{F}_{3}, \mathscr{F}_{4}\right)$, define $\mathscr{F}_{2}$ to be the natural inverse image of $m\left(\mathscr{F}_{3}\right) \subseteq \mathscr{F}_{4}$ in $c^{-1}\left(\mathscr{F}_{3}\right) \subseteq \mathscr{E}_{2}$. Let $\mathscr{R}_{2}=\mathscr{E}_{2} / \mathscr{F}_{2}$ and let $u_{2}: \mathscr{E}_{2} \rightarrow \mathscr{R}_{2}$ be the quotient homomorphism. Since $c\left(\mathscr{F}_{2}\right)=\bar{c} m\left(\mathscr{F}_{3}\right)=\mathscr{F}_{3}, c$ induces a surjection $t: \mathscr{R}_{2} \rightarrow \mathscr{R}_{3}$. Further $b\left(\mathscr{F}_{1}\right)=\mathscr{F}_{2} \cap b\left(\mathscr{E}_{1}\right)$ so that $b$ induces an inclusion $s: \mathscr{R}_{1} \rightarrow \mathscr{R}_{2}$. Then $(s, t)$ is exact and $u_{i}, 1 \leqq i \leqq 3, t, s$ fit into a commutative diagram (8), thus giving (an isomorphism class of) a completion of (4). Finally it is readily checked that the above correspondences $(8) \rightarrow j c_{0}$ 
and $m \rightarrow(8)$ are inverses to each other.

q. e. d.

b) Let $f: X \rightarrow S$ be a proper morphism of complex spaces with $S$ reduced. Suppose that we are given a diagram (4) on $X$. Let $\nu$ : $T \rightarrow S$ be any morphism of complex spaces. Then pulled back by $\nu$ (4) gives rise to the diagram

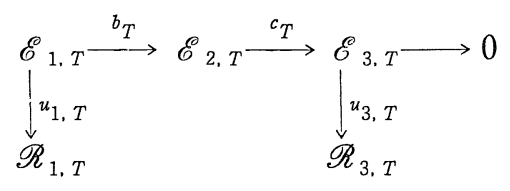

with the same property as (4). Then we set

$$
\left\{\begin{array}{l}
\mathscr{F}_{T, i}=\operatorname{Ker} u_{i, T}, \quad i=1,3 \\
\mathscr{F}_{T, 4}=c_{T}^{-1}\left(\mathscr{F}_{T, 3}\right) / b_{T}\left(\mathscr{F}_{T, 1}\right) .
\end{array}\right.
$$

Then we have the natural homomorphisms

$$
\left\{\begin{array}{l}
v_{i, T}: \mathscr{F}_{i, T} \longrightarrow \mathscr{F}_{T, i}, \quad i=1,3 \\
w_{T}: c^{-1}\left(\mathscr{F}_{3}\right)_{T} \longrightarrow c_{T}^{-1}\left(\mathscr{F}_{T, 3}\right)
\end{array}\right.
$$

where $v_{i, T}$ is surjective. Further $v_{1, T}$ and $w_{T}$ induce a homomorphism

$$
v_{4, T}: \mathscr{F}_{4, T} \longrightarrow \mathscr{F}_{T, 4}
$$

Lemma 8. Suppose that $\mathscr{E}_{3}$ and $\mathscr{R}_{3}$ are f-flat. Then $v_{3, T}$ and $v_{4, T}$ are isomorphic.

Proof. For $v_{3, T}$ this is immediate from the flatness of $\mathscr{R}_{3}$. We shall show that $v_{4, T}$ is isomorphic. Since $v_{1, T}$ is surjective, $b_{T}\left(\mathscr{F}_{T, 1}\right)=$ $b_{T} v_{1, T}\left(\mathscr{F}_{1, T}\right)$ in $\mathscr{E}_{2, T}$. Hence it suffices to show that $w_{T}$ is isomorphic. Let $\mathscr{K}^{T}$ be the kernel of $c_{T}: \mathscr{E}_{2, T} \rightarrow \mathscr{E}_{3 . T}$. Then since $\mathscr{E}_{3}$ is f-flat, the natural homomorphism $\mathscr{K}_{T} \rightarrow \mathscr{K}^{T}$ is isomorphic. The assertion then follows from the following commutative diagram (cf. (6))

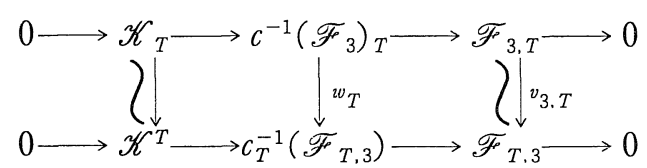

where the top sequence is exact since $\mathscr{F}_{3}$ is $f$-flat as well as $\mathscr{E}_{3}$ and $\mathscr{R}_{3}$.

q. e. d.

Suppose now that we are given a completion (8) of (4). We 
assume that $\mathscr{R}_{3}$ is $f$-flat. Then the pull-back $(8)_{T}$ of (8);

$(8)_{T}$

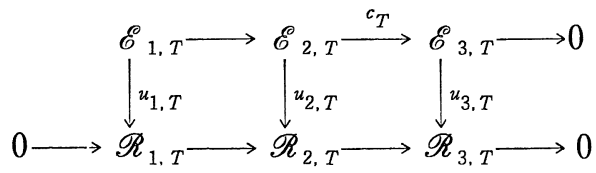

is again a completion of $(4)_{T}$ because $\mathscr{R}_{1, T} \longrightarrow \mathscr{R}_{2, T}$ is now injective. Thus we can speak of the functor $G:(\mathrm{An} / S)^{\circ} \longrightarrow$ Sets defined by $G(T):=$ the set of isomorphism classes of completions of $(4)_{T}$.

We set

$$
\mathscr{G}=\mathscr{H}_{a m 0_{X}}\left(\mathscr{F}_{3}, \mathscr{F}_{4}\right) \text { and } \mathscr{H}=\mathscr{H}_{a m 0_{X}}\left(\mathscr{F}_{3}, \mathscr{F}_{3}\right)
$$

where $\mathscr{F}_{3}$ and $\mathscr{F}_{4}$ are as in (5). Then the homomorphism $\alpha: \mathscr{G} \rightarrow$ $\mathscr{H}$ (cf. (7)) induces the natural homomorphism

$$
\beta: f_{*} \mathscr{G} \longrightarrow f_{*} \mathscr{H} \text {. }
$$

In order to have the representability of $G$ we make the following assumption ;

$$
\left\{\begin{array}{l}
\mathscr{E}_{3}, \mathscr{R}_{3}, \mathscr{G}, \mathscr{H} \text { are all } f \text {-flat, }\left(\mathscr{F}_{3}, \mathscr{F}_{4}\right) \text { and }\left(\mathscr{F}_{3}, \mathscr{F}_{3}\right) \\
\text { satisfy the condition }(\mathrm{C}) \text { over } S \text { and } \operatorname{dim} H^{0}\left(X_{s}, \mathscr{G}_{s}\right) \\
\text { and } \operatorname{dim} H^{0}\left(X_{s}, \mathscr{H}_{s}\right) \text { are locally constant on } S .
\end{array}\right.
$$

Since $S$ is reduced, this implies that

$$
f_{*} \mathscr{G} \text { and } f_{*} \mathscr{H} \text { are locally free on } S
$$

and further that for any $\nu: T \rightarrow S$ as above

$$
\left\{\begin{array}{l}
\text { the natural homomorphism } a_{T}: \nu^{*} f_{*} \mathscr{G} \longrightarrow f_{T *} \mathscr{G}_{T} \text { and } \\
a_{T}^{\prime}: \nu^{*} f_{*} \mathscr{H} \longrightarrow f_{T *} \mathscr{H}_{T} \text { are isomorphic (cf. [1]). }
\end{array}\right.
$$

By (11) we can consider the holomorphic vector bundle $M(\operatorname{resp} . N)$ associated to $f_{*} \mathscr{G}$ (resp. $\left.f_{*} \mathscr{H}\right) \quad(\operatorname{cf}$. $(1.1, b))$. Let $\gamma: M \rightarrow N$ be the bundle homomorphism corresponding to $\beta$. Let $\iota \in H^{0}(S, \mathscr{S}(N))$ correspond to $i d \in \operatorname{Hom}_{\mathcal{O}_{X}}\left(\mathscr{F}_{3}, \mathscr{F}_{3}\right)$ with respect to the natural isomorphism

$$
H^{0}(S, \mathscr{S}(N)) \cong H^{0}\left(S, f_{*} \mathscr{H}\right) \cong H^{0}(X, \mathscr{H}) \cong \operatorname{Hom}_{\mathcal{O}_{X}}\left(\mathscr{F}_{3}, \mathscr{F}_{3}\right)
$$

Define

$$
Y=\gamma^{-1}(\iota(S)) \text {. }
$$

Then $Y$ is an analytic subspace of $M$ and hence is naturally a complex space over $S$. 
Proposition 1. Under the assumption (10) $G$ is represented by the complex space $Y$ above.

Proof. Let $\nu: T \rightarrow S$ be any morphism of complex spaces. First note that since $\mathscr{E}_{3}$ and $\mathscr{R}_{3}$ are $f$-flat, $\mathscr{F}_{3}$ also is $f$-flat. Further by Lemma 6 the canonical homomorphism $\tilde{\nu}^{\sharp}: \mathscr{G}_{T} \rightarrow \mathscr{H}_{0 m \mathcal{O}_{X}}\left(\mathscr{F}_{3 . T}, \mathscr{F}_{4, T}\right)$ is isomorphic. Also, by Lemma $8 v_{3, T}$ and $v_{4, T}$ induce an isomorphism $d_{T}: \mathscr{H}_{m \mathcal{O}_{X}}\left(\mathscr{F}_{3, T}, \mathscr{F}_{4, T}\right) \rightarrow \mathscr{H}_{0 m \mathcal{O}_{X}}\left(\mathscr{F}_{T, 3}, \mathscr{F}_{T, 4}\right)$. Together with these induce a natural isomorphism $g_{T}: \nu^{*} f_{*} \mathscr{G} \rightarrow f_{T *} \mathscr{H}_{\left(a m . O_{X}\right.}\left(\mathscr{F}_{T .3}, \mathscr{F}_{T .4}\right)$. Similarly we get an isomorphism $h_{T}: \nu^{*} f_{*} \mathscr{H}_{\rightarrow} f_{T *} \mathscr{H}_{\text {om }_{X_{X}}}\left(\mathscr{F}_{T, 3}, \mathscr{F}_{T, 3}\right)$.

Using these isomorphisms for $T=Y$ we shall now construct the universal completion of $(4)_{Y}$ on $X_{Y}$. Let $\pi: M \rightarrow S$ be the natural projection and set $\delta=\left.\pi\right|_{Y}: Y \rightarrow S$. Let $\sigma \in H^{0}\left(Y, \delta^{*} f_{*} \mathscr{G}\right)$ be the restriction to $Y$ of the universal section $\tilde{\sigma} \in H^{0}\left(M, \pi^{*} f_{*} \mathscr{G}\right)$. Let $\sigma^{\prime}=$ $g_{Y}(\sigma) \in H^{0}\left(S, f_{Y *} \mathscr{H}_{o m \mathcal{O}_{X}}\left(\mathscr{F}_{Y, 3}, \mathscr{F}_{Y, 4}\right)\right) \cong \operatorname{Hom}_{\mathcal{O}_{X}}\left(\mathscr{F}_{Y, 3}, \mathscr{F}_{Y, 4}\right)$. Similarly, let $\iota^{\prime}=h_{Y}(\iota) \in \operatorname{Hom}_{\mathcal{O}_{X}}\left(\mathscr{F}_{Y, 3}, \mathscr{F}_{Y, 3}\right)$. Then $\iota^{\prime}$ is the identity of $\mathscr{F}_{Y, 3}$ and $\sigma^{\prime}$ is sent to $\ell^{\prime}$ by the natural homomorphism $\operatorname{Hom}_{\mathcal{O}_{X}}\left(\mathscr{F}_{Y, 3}, \mathscr{F}_{Y, 4}\right) \rightarrow$ Hom $_{\mathcal{O}_{Y}}\left(\mathscr{F}_{Y, 3}, \mathscr{F}_{Y, 3}\right)$. Therefore by Lemma $7 \sigma^{\prime}$ gives rise to a completion (14) of $(4)_{Y}$;

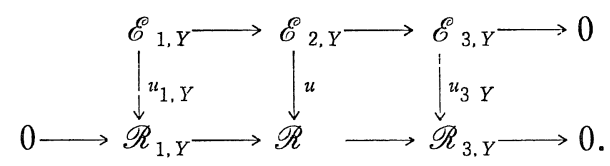

We claim that (14) is the desired universal completion (up to isomorphisms). Let $\nu: T \rightarrow S$ be any morphism of complex spaces with a completion $(8)_{T}$ of $(4)_{T}$. Then by Lemma $7(4)_{T}$ defines an element $\lambda^{\prime} \in \operatorname{Hom}_{\mathcal{O}_{X}}\left(\mathscr{F}_{T, 3}, \mathscr{F}_{T, 4}\right)$ whose image in $\operatorname{Hom}_{\mathcal{O}_{X}}\left(\mathscr{F}_{T .3}, \mathscr{F}_{T .3}\right)$ is the identity. Let $\lambda=g_{T}^{-1}\left(\lambda^{\prime}\right) \in H^{0}\left(T, \nu^{*} f_{*} \mathscr{G}\right)$. Then the image of $\lambda$ in $H^{0}\left(X, \nu^{*} f_{*} \mathscr{H}\right)$ by $\nu^{*}(\beta)$ equals $\nu^{*}(\iota)$. Hence by the universality of $M$ together with the definition of $Y$ there exists a unique $S$ morphism $\tau: T \rightarrow Y$ such that $\lambda$ is the pull-back of $\sigma$ by $\tau ; \lambda=\tau^{*}(\sigma)$. This then implies that $\tau^{*}\left(\sigma^{\prime}\right)=\lambda^{\prime}$ with respect to the natural isomorphism $\tilde{\tau}^{*} \mathscr{H}_{o m 0_{X}}\left(\mathscr{F}_{Y, 3}, \mathscr{F}_{Y, 4}\right) \cong \mathscr{H}_{a m 0_{X}}\left(\tilde{\tau}^{*} \mathscr{F}_{Y, 3}, \tilde{\tau}^{*} \mathscr{F}_{Y, 4}\right) \cong \mathscr{H}_{a m 0_{X}}\left(\mathscr{F}_{T, 3}\right.$, $\mathscr{F}_{T, 4}$ ), where $\tilde{\tau}: X_{T} \cong X_{Y} \times_{Y} T \rightarrow X_{Y}$ is the natural projection (cf. Lemmas 6 and 8 ). This in turn is equivalent to saying that $(8)_{T}$ is isomorphic 
to the pull-back of (14) by $\tau$, in view of the correspondence of Lemma 7. q. e. d.

1.3. Let $f: X \rightarrow S$ be a proper morphism of complex spaces with $S$ reduced. Suppose that we are given a diagram (4) on $X$. As in 1.2 b) we set $\mathscr{G}=\mathscr{H}_{\mathrm{m}_{\mathcal{O}_{X}}}\left(\mathscr{F}_{3}, \mathscr{F}_{4}\right)$ and $\mathscr{H}=\mathscr{H}_{\mathrm{m} \mathcal{O}_{X}}\left(\mathscr{F}_{3}, \mathscr{F}_{3}\right)$ where $\mathscr{F}_{3}, \mathscr{F}_{4}$ are defined by (5). We fix a Zariski open subset $U \subseteq S$ with the following property (cf. Lemma 5);

$$
\left\{\begin{array}{l}
\mathscr{E}_{3}, \mathscr{R}_{3}, \mathscr{G}, \mathscr{H} \text { are all } f \text {-flat over } U,\left(\mathscr{F}_{3}, \mathscr{F}_{4}\right) \text { and }\left(\mathscr{F}_{3}, \mathscr{F}_{3}\right) \\
\text { satisfy the condition (C) over } U \text {, and } \operatorname{dim} H^{0}\left(X_{s}, \mathscr{G}_{s}\right) \text { and } \\
\operatorname{dim} H^{0}\left(X_{s}, \mathscr{H}_{s}\right) \text { are locally constant on } U .
\end{array}\right.
$$

We then apply the consideration of 1.2 b) to $f_{U}: X_{U} \rightarrow U$ and (4) the restriction of (4) over $U$. Let $M_{0}$ and $N_{0}$ be the vector bundles on $U$ associated to the locally free sheaves $\left.f_{*} \mathscr{G}\right|_{U}$ and $\left.f_{*} \mathscr{H}\right|_{U}$ respectively. Let $\gamma: M_{0} \rightarrow N_{0}$ be the bundle homomorphism induced by $\beta: f_{*} \mathscr{G} \rightarrow f_{*} \mathscr{H}$ restricted to $U$ (cf.(9)). Let $\iota: U \rightarrow N_{0}$ be the holomorphic section defined by the identity of $\mathscr{F}_{3}$ via (13) for $S=U$. Let $Y=\gamma^{-1}(\iota(U)) \subseteq M_{0}$ and $\delta: Y \rightarrow U$ the natural morphism. Then by Proposition $1 Y$ represents the functor $G_{U}:(\mathrm{An} / U)^{\circ} \rightarrow$ Sets defined by $G_{U}\left(U^{\prime}\right)=$ the set of isomorphism classes of completions of $(4)_{T}$ with $T=U^{\prime}$. Thus we get the universal completion (14) on $Y$ (with $S$ replaced by $U$ there).

Proposition 2. In the above notations there exist a projective morphism $\bar{\delta}: \bar{Y} \rightarrow S$ and an inclusion $Y \subseteq \bar{Y}_{U}$ over $U$ such that $Y$ is a dense Zariski open subset of $\bar{Y}$. Moreover exists a coherent quotient $\bar{u}: \mathscr{E}_{2, \bar{Y}} \rightarrow \overline{\mathscr{R}}$ on $X_{\bar{Y}}=X \times{ }_{s} \bar{Y}$ such that the restriction of $\bar{u}$ to $X_{Y}=X \times{ }_{S} Y$ is isomorphic to the quotient $u: \mathscr{E}_{2, Y} \rightarrow \mathscr{R}$ in the universal completion (14) on $X_{Y}$.

Proof. a) The construction of $\bar{Y}$. Let $\mathscr{M}=f_{*} \mathscr{G}$ and $\mathscr{N}=f_{*} \mathscr{H}$. Let $L=L\left(\mathscr{M}^{*}\right) \quad$ (resp. $L^{\prime}=L\left(\mathscr{N}^{*}\right)$ ) be the linear fiber space over $S$ associated to the dual $\mathscr{M}^{*}\left(\right.$ resp. $\left.\mathscr{N}^{*}\right)$ of $\mathscr{M}($ resp. $\mathscr{N})$ (cf. 1.1 b)). Since $\mathscr{M} \cong \mathscr{M}^{* *}$ on $U$, we may identify $M_{0}$ with $L_{U}$. Let $\boldsymbol{P}=$ $\boldsymbol{P}\left(\mathscr{M}^{*} \oplus \mathcal{O}_{S}\right)$ (resp. $\left.\mathbb{F}^{\prime}=\boldsymbol{P}\left(\mathscr{N}^{*} \oplus \mathcal{O}_{S}\right)\right)$ be the projective fiber space over $S$ associated to $\mathscr{M}^{*} \oplus \mathcal{O}_{S}$ (resp. $\mathscr{N}^{*} \oplus \mathcal{O}_{S}$ ). Then we have the natural inclusions $L \subseteq \boldsymbol{P}$ and $L \subseteq \boldsymbol{P}^{\prime}$ as Zariski open subsets (cf. Lemma 
3). Then the transpose $\alpha^{*}: \mathscr{N}^{*} \rightarrow \mathscr{M}^{*}$ of $\alpha$ (cf. (7)) induces a homomorphism $b: L \rightarrow L^{\prime}$ of linear fiber spaces over $S$ which is easily seen to extend to a meromorphic map $\tilde{b}: \boldsymbol{P} \rightarrow \mathbb{P}^{\prime}$ over $S$ (cf. the proof of Lemma 3 and [8]). Let $\bar{\imath} \in H^{0}(S, \mathscr{S}(L))$ be the section induced by the identity of $\mathscr{F}_{3}$ via the natural homomorphism $\operatorname{Hom}_{\mathcal{O}_{X}}\left(\mathscr{F}_{3}, \mathscr{F}_{3}\right)$

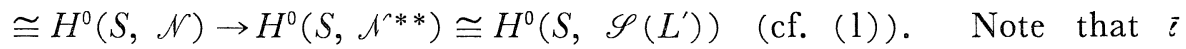
restricts to $\iota$ on $U$ with respect to the natural isomorphism $\mathscr{N} \cong \mathcal{I}^{* * *}$ on $U$. Let $\bar{Y}^{\prime} \cong \tilde{b}^{-1}(\bar{\iota}(S))$. Then we define $\bar{Y}$ to be the analytic closure of (i. e., the minimal analytic subspace containing) $Y=\bar{Y}_{U}^{\prime} \cap L_{U}$ in $\bar{Y}^{\prime}$. Then $Y$ is a dense Zariski open subset of $\bar{Y}$ and the natural morphism $\bar{\delta}: \bar{Y} \rightarrow S$ is projective as well as $\mathbb{P} \rightarrow S$.

b) Consinuction of a coherent quotient $\bar{u}: \mathscr{E}_{2, \bar{Y}} \rightarrow \overline{\mathscr{R}}$. Consider the diagram

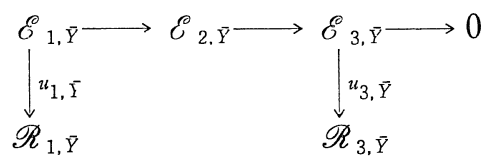

The restriction of $(4)_{\bar{Y}}$ to $X_{Y}$ admits the natural completion, i.e., the universal completion (14). Hence by Lemma 7, (14) determines a unique section $\lambda_{0}$ of $\mathscr{H}_{\text {om } \mathcal{O}_{X_{\bar{Y}}}}\left(\mathscr{F}_{\bar{Y}, 3}, \mathscr{F}_{\bar{Y}, 4}\right)$ on $X_{Y}$ (cf. $(5)_{T}$ for $\left.\mathscr{F}_{\bar{Y}, i}\right)$.

We first show that this $\lambda_{0}$ is extended meromorphically to the whole $X_{\bar{Y}}$. Clearly, it suffices to show that the holomorphic section $\lambda_{1}$ of $f_{\bar{Y} *} \mathscr{H}_{0 m \mathcal{O}_{X_{\bar{Y}}}}\left(\mathscr{F}_{\bar{Y}, 3}, \mathscr{F}_{\bar{Y}, 4}\right)$ on $Y$ determined by $\lambda_{0}$ is extended meromorphically to the whole $\bar{Y}$. In view of Lemma 2, for this purpose we have only to show the following assertions.

1) $\left(\bar{\delta}^{*} \mathscr{l l}^{*}\right)^{*}$ and $f_{\bar{Y} *} \mathscr{H}_{\text {orr }} \mathcal{O}_{X_{\bar{Y}}}\left(\mathscr{F}_{\bar{Y}, 3}, \mathscr{F}_{\bar{Y}, 4}\right)$ are meromorphically equivalent and isomorphic on $Y$, and

2) the section $\lambda_{2} \in H^{0}\left(Y,\left(\bar{\delta}^{*} \mathscr{H}^{*}\right)^{*}\right)$ corresponding to $\lambda_{1}$ by 1$)$ is extended meromorphically to $\bar{Y}$.

Proof of 1). Let $d_{1}: \bar{\delta}^{*} \mathscr{M} \rightarrow \bar{\delta}^{*}\left(\mathscr{M}^{* *}\right)$ be induced by the natural homomorphism $\mathscr{M} \rightarrow \mathscr{M}^{* *}$. Let $d_{2}: \bar{\delta}^{*}\left(\mathscr{l l}^{* *}\right) \rightarrow\left(\bar{\delta}^{*} \mathscr{M}^{*}\right) *$ be the canonical homomorphism (3) applied to $\mathscr{F}=\mathscr{M}^{*}$. Let $d_{3}=a_{\bar{Y}}: \bar{\delta}^{*} \mathscr{M}=\bar{\delta}^{*} f_{*} \mathscr{G} \rightarrow$ $f_{\bar{Y} *} \mathscr{G}_{\bar{Y}}$ (cf. (12)). Let $d_{4}: f_{\bar{Y} *} \mathscr{G}_{\bar{Y}} \rightarrow f_{\bar{Y} *}\left(\mathscr{H}_{a m 0_{X_{\bar{Y}}}}\left(\mathscr{F}_{3, \bar{Y}}, \mathscr{F}_{4, \bar{Y}}\right)\right)$ be induced by the canonical homomorphism (2) $\mathscr{G}_{\bar{Y}}=\mathscr{H}_{\theta_{0} \theta_{X}}\left(\mathscr{F}_{3}, \mathscr{F}_{4}\right)_{\bar{Y}} \rightarrow$ $\mathscr{H}_{\text {om } \mathcal{O}_{\bar{Y}}}\left(\mathscr{F}_{3, \bar{Y}}, \mathscr{F}_{4, \bar{Y}}\right)$. These $d_{i}$ are all isomorphic on $Y$ (cf. Lemma 6). Hence $\left(\bar{\delta}^{*} \mathscr{l}^{*}\right)^{*}$ and $f_{\bar{Y} *}\left(\mathscr{H}_{0 m \sigma_{\bar{Y}} \bar{Y}}\left(\mathscr{F}_{3, \bar{Y}}, \mathscr{F}_{4, \bar{Y}}\right)\right)$ are meromorphically 
equivalent and isomorphic on $Y$. Next note that $v_{i, \bar{Y}}: \mathscr{F}_{i, \bar{Y}} \rightarrow \mathscr{F}_{\bar{Y}, i}$, $i=3$, 4, are isomorphic on $X_{Y}$ (cf. Lemma 8). Then $v_{3, \bar{Y}}$ and $v_{4, \bar{Y}}$ induce homomorphisms $f_{\bar{Y} *}\left(\mathscr{H}_{o m \theta_{X_{\bar{Y}}}}\left(\mathscr{F}_{\bar{Y}, 3}, \mathscr{F}_{4, \bar{Y}}\right)\right) \rightarrow f_{\bar{Y} *}\left(\mathscr{H}_{\text {tom }}{ }_{X_{\bar{Y}}}\left(\mathscr{F}_{\bar{Y}, 3}\right.\right.$, $\left.\left.\mathscr{F}_{\bar{Y}, 4}\right)\right)$ and $f_{\bar{Y} *}\left(\mathscr{H}_{0 m \theta_{X_{\bar{Y}}}}\left(\mathscr{F}_{\bar{Y}, 3}, \mathscr{F}_{4, \bar{Y}}\right)\right) \rightarrow f_{\bar{Y} *}\left(\mathscr{H}_{0 m \theta_{X_{\bar{Y}}}}\left(\mathscr{F}_{\bar{Y}, 3}, \mathscr{F}_{\bar{Y}, 4}\right)\right)$ respectively which are isomorphic on $Y$. Hence $f_{\bar{Y} *}\left(\mathscr{H}_{0 m O_{X_{\bar{Y}}}}\left(\mathscr{F}_{\bar{Y}, 3}, \mathscr{F}_{\bar{Y}, 4}\right)\right)$ and $f_{\bar{Y} *}\left(\mathscr{H}_{a m \mathcal{O}_{X_{\bar{Y}}}}\left(\mathscr{F}_{3, \bar{Y}}, \mathscr{F}_{4, \bar{Y}}\right)\right)$, and hence the former and $\left(\bar{\delta}^{*} \mathscr{M}^{*}\right)^{*}$ also, are meromorphically equivalent and isomorphic on $Y$. Thus 1) is proved.

Proof of 2). Let $\pi: L \rightarrow S$ and $\bar{\pi}: \boldsymbol{P} \rightarrow S$ be the natural projections. Consider the following commutative diagram of coherent analytic sheaves on $\boldsymbol{P}$

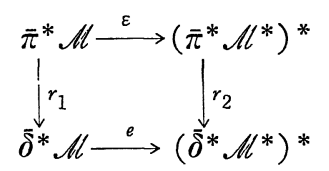

which is isomorphic on $M_{0}$, where $e=d_{2} d_{1}$ and $\varepsilon$ is defined analogously and where the vertical arrows are induced by the inclusion $\bar{Y} \subseteq \boldsymbol{P}$. This gives rise to a commutative diagram of the spaces of global sections

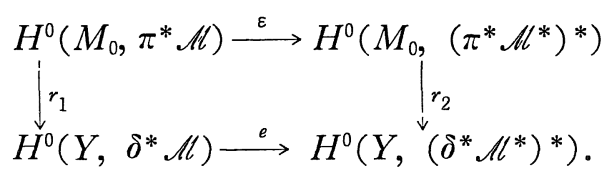

Let $\tilde{\sigma} \in H^{0}\left(M_{0}, \pi^{*} \mathscr{M}\right)$ be the universal section. Then $r_{2} \varepsilon(\tilde{\sigma})=e r_{1}(\tilde{\sigma})=$ $\lambda_{2}$ (cf. the proof of Proposition 1). Thus it suffices to show that $\varepsilon(\tilde{\sigma})$ is extended meromorphically to the whole $\boldsymbol{P}$. This, however, follows from Lemma 3 applied to $\mathscr{E}=\mathscr{M}^{*}$ since $\varepsilon(\tilde{\boldsymbol{\sigma}})$ is the restriction to $M_{0}$ of the universal section of $\left(\pi^{*} \mathscr{M}^{*}\right)^{*}$ on $L$. This proves 2).

Next, using the fact that $\lambda_{0}$ is extended meromorphically to $X_{\bar{Y}}$ we shall construct a desired quotient $\bar{u}: \mathscr{E}_{2, \bar{Y}} \rightarrow \overline{\mathscr{R}}$. Let $h: \tilde{X} \rightarrow X_{\bar{Y}}$ be the blowing up of $X_{\bar{Y}}$ with center $A:=X_{\bar{Y}}-X_{Y}$, where $A$ is endowed with the reduced structure. Then there exists an effective Cartier divisor $D$ on $\tilde{X}$ whose support coincides with $\tilde{X}-h^{-1}\left(X_{Y}\right)$. Since $h$ is isomorphic on $h^{-1}\left(X_{Y}\right)$ we identify $h^{-1}\left(X_{Y}\right)$ with $X_{Y}$ and consider $X_{Y}$ also as a Zariski open subset of $\tilde{X}$. Now consider the pull-back 
of $(4)_{\bar{Y}}$ to $\tilde{X}$ by $h$;

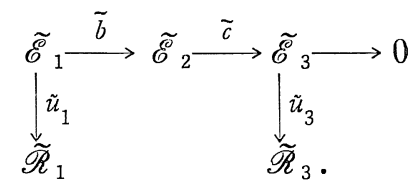

Let $\tilde{\mathscr{F}}_{i}=\operatorname{Ker} \tilde{u}_{i}, i=1,3$, and $\widetilde{\mathscr{F}}_{4}=\tilde{c}^{-1}\left(\tilde{\mathscr{F}}_{3}\right) / \tilde{b}\left(\widetilde{\mathscr{F}}_{1}\right)$. Then by the same argument as in the proof of 1$)$ we see readily that $h^{*} \mathscr{H}_{\text {am }}{ }_{X_{\bar{Y}}}\left(\mathscr{F}_{\bar{Y}, 3}\right.$, $\left.\mathscr{F}_{\bar{Y}, 4}\right)$ and $\mathscr{H}_{\mathrm{m}_{0} \mathcal{O}_{\tilde{X}}}\left(\widetilde{\mathscr{F}}_{3}, \widetilde{\mathscr{F}}_{4}\right)$ are meromorphically equivalent and isomorphic over $X_{Y}$. Thus the section $\tilde{\lambda}$ of $\mathscr{H}_{\text {omo }}\left(\tilde{\mathscr{F}}_{3}, \tilde{\mathscr{F}}_{4}\right)$ on $X_{Y}$ corresponding to $h^{*} \lambda_{0}$ is extended meromorphically to $\tilde{X}$ by Lemma 2 . Hence by Lemma 1 for any relatively compact subdomain $W \subseteq \tilde{X}$ we can find an integer $n>0$ and a holomorphic section $\tilde{\lambda}^{*}=\tilde{\lambda}_{W}^{*}$ of $\mathscr{H}_{\text {fom }{ }_{\tilde{X}}}\left(\tilde{\mathscr{F}}_{3}, \widetilde{\mathscr{F}}_{4}\right)(n D)$ on $\tilde{W}:=h^{-1}(I V)$ such that $\tilde{\lambda}^{*}$ restricts to $\tilde{\lambda}_{\tilde{W} \cap X_{Y}}$ in the obvious sense. Then through the natural isomorphism $\mathscr{H}_{\text {omo }}\left(\tilde{\mathscr{F}}_{3}, \tilde{\mathscr{F}}_{4}\right)(n D) \cong \mathscr{H}_{\text {om }}\left(\tilde{\mathscr{F}}_{3}(-n D), \tilde{\mathscr{F}}_{4}\right), \tilde{\lambda}^{*}$ determines a homomorphism $\hat{\lambda}^{*}: \tilde{\mathscr{F}}_{3}(-n D) \rightarrow \tilde{\mathscr{F}}_{4}$ on $\tilde{W}$. Let $\tilde{\mathscr{F}}(W)=\zeta^{-1}\left(\hat{\lambda}^{*}\left(\tilde{\mathscr{F}}_{3}(-n D)\right)\right)$ where $\zeta: \tilde{c}^{-1}\left(\tilde{\mathscr{F}}_{3}\right) \rightarrow \tilde{\mathscr{F}}_{4}$ is the natural homomorphism. Then if we set $\mathscr{F}=\operatorname{Ker} u, \tilde{\mathscr{F}}(W)=\mathscr{F}$ on $X_{Y} \cap \tilde{W}$ as a submodule of $\widetilde{\mathscr{E}}_{2}$. Further $h_{*} \tilde{\mathscr{F}}(W)$ is a coherent submodule of $h_{*} \widetilde{\mathscr{E}}_{2}=h_{*} h^{*} \mathscr{E}_{2, \bar{Y}}$. Let $a: \mathscr{E}_{2, \bar{Y}} \rightarrow$ $h_{*} h^{*} \mathscr{E}_{2, \bar{Y}}$ be the natural homomorphism which is isomorphic on $X_{Y}$. Then $\overline{\mathscr{F}}(W):=a^{-1}\left(h_{*} \tilde{\mathscr{F}}(W)\right)$ is a coherent submodule of $\mathscr{E}_{2, \bar{Y}}$ on $W$ with $\left.\overline{\mathscr{F}}(W)\right|_{W \cap X_{Y}}=\left.\mathscr{F}\right|_{W \cap X_{Y^{*}}}$ Thus we have shown that $\mathscr{F}$ extends locally to a coherent submodule of $\mathscr{E}_{2, \bar{Y}}$ at any point of $\bar{Y}$.

Now we define the submodule $\mathscr{F}\langle A\rangle$ of $\mathscr{E}_{2, \bar{Y}}$, which is defined on the whole $X_{\bar{Y}}$ and which extends $\mathscr{F}$, by the following condition; a holomorphic section $s$ of $\mathscr{E}_{2, \bar{Y}}$ defined on an open subset $B$ of $X_{\bar{Y}}$ is a section of $\mathscr{F}\langle A\rangle$ if and only $\left.s\right|_{X_{Y} \cap B} \in H^{0}\left(X_{Y} \cap B, \mathscr{F}\right)$. Since on any $W$ as above $\mathscr{F}\langle A\rangle=\left(\left.\overline{\mathscr{F}}(W)\right|_{x_{Y}}\right)\langle A\rangle$ (with the right hand side defined in the same way) and $\overline{\mathscr{F}}(W)$ is coherent on $W$ it follows that $\mathscr{F}\langle A\rangle$ is a coherent submodule of $\mathscr{E}_{2, \bar{Y}}$ on the whole $X_{\bar{Y} \bullet}$ (On $W, \mathscr{F}\langle A\rangle$ is characterized by the exact sequence $0 \rightarrow \overline{\mathscr{F}}(W) \rightarrow \mathscr{F}\langle A\rangle \rightarrow$ $\mathscr{H}_{A}^{0}\left(\mathscr{E}_{2, \bar{Y}} / \overline{\mathscr{F}}(W)\right) \rightarrow 0$.) Thus if we set $\overline{\mathscr{R}}=\mathscr{E}_{2, \bar{Y}} / \mathscr{F}\langle A\rangle$, then the natural homomorphism $\bar{u}: \mathscr{E}_{2, \bar{Y}} \rightarrow \overline{\mathscr{R}}$ satisfies the condition of the proposition. q.e.d.

1.4. Let $f: X \rightarrow B$ be a proper morphism of complex spaces. Let $m$ 
$\geqq 1$ be an integer. Suppose that for each $0 \leqq k \leqq m$ we are given coherent analytic sheaves $\mathscr{E}_{k}, \mathscr{E}^{k}$, and a coherent quotient $u_{k}: \mathscr{E}_{k} \rightarrow$ $\mathscr{R}_{k}$ on $X$ such that 1) $\mathscr{E}^{0}=\mathscr{E}_{0}$ and 2) $\mathscr{E}_{k}, \mathscr{E}^{k}$ fit into an exact sequence

$$
\mathscr{E}_{k} \longrightarrow \mathscr{E}^{k} \longrightarrow \mathscr{E}^{k-1} \longrightarrow 0, \quad k \geqq 1 .
$$

Let $U$ be a complex variety and $\tau: U \rightarrow B$ be a morphism of complex spaces. Suppose that for each $1 \leqq k \leqq m$ there exists a commutative diagram of exact sequences

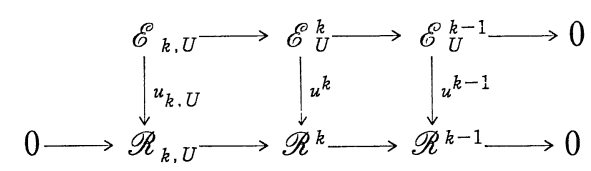

of coherent analytic sheaves on $X_{U}$ with all $u^{k}$ surjective as well as $u_{k, U}$, where $\left(u^{0}: \mathscr{E}_{U}^{0} \rightarrow \mathscr{R}^{0}\right)=\left(u_{0, U}: \mathscr{E}_{U}^{0} \rightarrow \mathscr{R}_{0, U}\right)$ by definition. Then we have the following:

Lemma 9. We can find 1) a complex variety $A$ which is locally projective over $B(c f .[4,(1.2)]), 2)$ a dense open subset $V$ of $U$ and a $B$-morphism $\eta: V \rightarrow A$ and 3) a coherent quotient $u: \mathscr{E}_{A}^{m} \rightarrow \mathscr{R}$ of $\mathscr{E}_{A}^{m}$, such that a) the pull-back of u to $X_{V}=X_{A} \times_{A} V$ via $\eta$ is isomorphic to the restriction of $u^{m}: \mathscr{E}_{U}^{m} \rightarrow \mathscr{R}^{m}$ to $X_{V}$ and $\left.b\right) A$ is the analytic closure of $\eta(V)$ in $A$.

Proof. Replacing $B$ by the analytic closure $B_{0}$ of $\tau(U)$ we may assume that $B=B_{0}$. In particular $B$ is reduced. Now we prove the lemma by induction on $m \geqq 1$. So suppose that the lemma is true for the data above with $0 \leqq k \leqq m-1$. Then we can find a complex variety $A^{\prime}$ which is locally projective over $B$, a dense open subset $V^{\prime} \subseteq U$, a $B$-morphism $\eta^{\prime}: V^{\prime} \rightarrow A^{\prime}$ and a coherent quotient $u^{\prime}: \mathscr{E}_{A^{\prime}}^{m-1} \rightarrow$ $\mathscr{R}^{\prime}$ of $\mathscr{E}_{A^{\prime}}^{m-1}$ such that a) the pull-back of $u^{\prime}$ via $\eta^{\prime}$ is isomorphic to $u^{m-1}$ restricted to $X_{V^{\prime}}$ and b' the analytic closure of $\eta^{\prime}\left(V^{\prime}\right)$ in $A^{\prime}$ coincides with $A^{\prime}$. Here, when $m=1$ (the beginning of the induction) we set $V^{\prime}=U, A^{\prime}=B, \eta^{\prime}=\tau$ and $u^{\prime}=u_{0}$. Now we consider the diagram

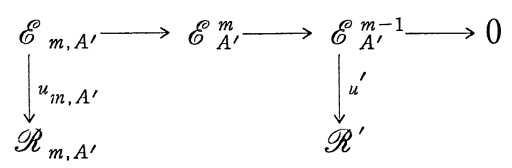


Let $U^{\prime}$ be a Zariski open subset of $A^{\prime}$ which satisfies the condition (15) for $f_{A^{\prime}}: X_{A^{\prime}} \rightarrow A^{\prime}$ and for (17) (instead of $f: X \rightarrow S$ and (4)). Let $Y^{\prime}$ be the complex space over $U^{\prime}$ which represents the functor $G_{U^{\prime}}:\left(\mathrm{An} / U^{\prime}\right)^{\circ} \rightarrow$ Sets $; G_{U^{\prime}}\left(U^{\prime \prime}\right)=$ the set of isomorphism classes of completions of (17) $)_{J^{\prime \prime}}\left(\mathrm{cf}\right.$. Proposition 1). Then $X_{Y^{\prime}}=X_{U^{\prime}} \times_{U^{\prime}} Y^{\prime}$ carries the universal completion

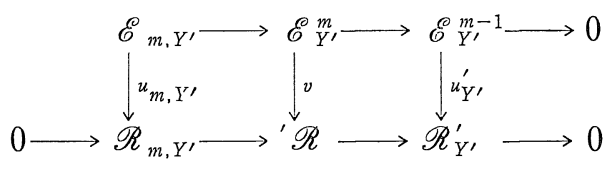

of $(17)_{Y^{\prime}}$. Let $\delta: Y^{\prime} \rightarrow U^{\prime}$ be the natural morphism. Let $\bar{\delta}: \bar{Y} \rightarrow A^{\prime}$ be the projective morphism which extends $\delta$ obtained in Proposition 2. By that proposition $v$ extends to a coherent quotient $\bar{v}: \mathscr{E}_{Y^{\prime}} \rightarrow \rightarrow^{\prime} \overline{\mathscr{R}}$ on $X_{\bar{Y} \prime}$. Let $V=\eta^{\prime-1}\left(U^{\prime}\right)$. Then by b) $V$ is dense in $V^{\prime}$ and hence also in $U$. On the other hand, by a) $(16)_{m}$ (restricted over $V^{\prime}$ ) is regarded as a completion of the pull-back $(17)_{V^{\prime}}$ of (17) to $X_{V^{\prime}}$ via $\eta^{\prime}$. Hence by the universality of $Y^{\prime},\left.\eta^{\prime}\right|_{V}$ lifts to a unique morphism $\eta: V \rightarrow Y^{\prime}$ such that the pull-back of (18) to $X_{V}$ via $\eta$ is isomorphic to (16) ${ }_{m, V}$. Thus if we define $A$ by the analytic closure of $\eta(V)$ in $\bar{Y}^{\prime}$ and $u: \mathscr{E}_{A}^{m} \rightarrow \mathscr{R}$ by the restriction of $\bar{v}$ on $X_{A}$, then the conditions a) and b) are obviously fulfilled (cf. [4, (1.2.1)] for the local projectivity).

q. e. d.

Remark 1. Actually we can show that $\eta$ is a restriction of a meromorphic map $U \rightarrow A$ over $B$, and hence we can take the above $V$ as a Zariski open subset of $U$.

\section{§ 2. Proof of Theorem}

2.1. We begin with two lemmas which are used in [3] without explicit proof.

Lemma 10. Let $g: Z \rightarrow T$ be a proper flat morphism of complex spaces. Suppose that $Z$ is (i.e., $Z_{\mathrm{red}}$ is) pure dimensional and $T$ is irreducible. Let $\mathscr{F}$ be a g-flat coherent analytic sheaf on $X$. Let $T^{\prime}$ be a complex variety and $\eta: T^{\prime} \rightarrow T$ a morphism. Then the followings hold true. 1) If $Z_{T^{\prime}}$ is reduced, then $Z$ itself is reduced. If, further, $Z_{T^{\prime}}$ is 
irreducible, then so is $Z$. 2) Suppose that $Z_{T}$, is reduced and $\mathscr{F}_{T}$, is torsion free. Then $\mathscr{F}$ also is torsion free.

Proof. Write $Z^{\prime}=Z_{T^{\prime}}$ and $\mathscr{F}^{\prime}=\mathscr{F}_{T^{\prime}}$. Let $g^{\prime}: Z^{\prime} \rightarrow T^{\prime}$ be the natural morphism. Suppose first that $Z^{\prime}$ is reduced. Since $T^{\prime}$ is a variety and $g^{\prime}$ is flat, $Z^{\prime}$ is pure dimensional as well as $Z$. Then by 3 ) $\rightarrow$ 1) of [3, Lemma 1.4] $Z_{t^{\prime}}^{\prime}$ is reduced for some $t^{\prime} \in T^{\prime}$ and hence $Z_{\eta\left(t^{\prime}\right)}$ also is reduced. Then by 1$) \rightarrow 3$ ) of the same lemma $Z$ is reduced. If further $\mathscr{F}^{\prime}$ is torsion free, then by 3$) \rightarrow 1$ ) of $[3$, Lemma 5.6] $\mathscr{F}^{\prime \prime}$ is a torsion free $\mathcal{O}_{\left.z_{\eta(t}\right)}$-module for some $t^{\prime \prime} \in T^{\prime}$. Then by 1$) \rightarrow$ 3 ) of the same lemma $\mathscr{F}$ is torsion free.

Next suppose that $Z^{\prime}$ is reduced and irreducible. Let $t^{\prime} \in T^{\prime}$ be as above and set $t=\eta\left(t^{\prime}\right)$ so that $Z_{t}$ is reduced. Let $Z_{1}, \ldots, Z_{r}$ be the irreducible components of $Z$. We have to show that $r=1$. We use the same argument as in [4, Prop. 3]. By our assumption, for any $i Z_{i} \supseteqq Z_{t}$, and hence $Z_{i, t}=Z_{t}$, since $g\left(Z_{i}\right)=T$. Let $Z_{i j}=Z_{i} \cap Z_{j}$ and $T_{i j}=\left\{t \in T ; \operatorname{dim} Z_{i j, t} \geqq \operatorname{dim} Z-\operatorname{dim} T\right\}$. Let $T_{0}=\cup_{i \neq j} T_{i j}$. Then we can take a holomorphic map $h: H \rightarrow T$ of the unit disc $H:=$ $\{d \in \boldsymbol{C} ;|d|<1\}$ into $T$ such that $h(0)=t$ and $h^{-1}\left(T_{0}\right)=\{0\}$. Set $\tilde{Z}=$ $Z_{H}$ and $\tilde{Z}_{i}=Z_{i, H}$. Then by [3, Lemma 1.4] $\tilde{Z}$, and hence $\tilde{Z}_{i}$ also, are reduced since $\tilde{Z}_{0}$ is reduced. Hence $\tilde{Z}_{i} \rightarrow H$ is flat as well as $\tilde{Z} \rightarrow H$. Let $\chi$ be any Hermitian form on $\tilde{Z}$ (cf. [3, Def. 1.2]) and $\chi_{i}$ the restriction of $\chi$ to $Z_{i}$. Then by [3, Cor. 3.3] the positive functions

$$
\lambda(d):=\int_{\tilde{z}_{d}} \chi_{d}, \quad \lambda_{i}(d):=\int_{\tilde{z}_{i, d}} \chi_{i, d}
$$

are continuous on $H$. (Note that by taking $h$ suitably, we may assume that $\tilde{Z}_{d}$ and $\tilde{Z}_{i, d}$ are all reduced for $d \in H$.) Hence

$$
\begin{gathered}
\lim _{d \rightarrow 0} \sum_{i=1}^{r} \lambda_{i}(d)=\sum_{i=1}^{r} \lambda_{i}(0)=\sum_{i=1}^{r} \int_{\tilde{z}_{i, 0}} \chi_{i, 0} \\
=r \int_{\tilde{z}_{0}} \chi_{0}=r \lambda(0)=r \lim _{d \rightarrow 0} \lambda(d) .
\end{gathered}
$$

On the other hand, by our choice of $h, \lambda(d)=\sum_{i=1}^{r} \lambda_{i}(d)$ for any $d \neq 0$ 。 This is possible only when $r=1$. q. e. d.

Lemma 11. Let $f: X \rightarrow S$ be a morphism of complex spaces and $Z$ a subspace of $X$. Let $\mathscr{E}$ be a coherent analytic sheaf on $X$. Let $\bar{\psi}: T \rightarrow S$ 
be a morphism of complex spaces. Let $\mathscr{I}$ be the ideal sheaf of $Z$ in $X$ and $\mathscr{I}_{T}$ that of $Z_{T}$ in $X_{T}$. Then for each $k \geqq 0$ there exist a natural isomorphism $\lambda_{k}: \psi^{*}\left(\mathscr{E} / \mathscr{I}^{k} \mathscr{E}\right) \rightarrow \phi^{*} \mathscr{E} / \mathscr{I}_{T}^{k} \psi^{*} \mathscr{E}$ and a natural surjective homomorphism $\mu_{k}: \psi^{*}\left(\mathscr{I}^{k} \mathscr{E} / \mathscr{I}^{k+1} \mathscr{E}\right) \rightarrow \mathscr{I}_{T}^{k} \phi^{*} \mathscr{E} / \mathscr{I}_{T}^{k+1} \phi^{*} \mathscr{E}$, where $\phi: X_{T}$ $\rightarrow X$ is the natural projection.

Proof. First, $\lambda_{k}$ is defined by the requirement that the following diagram of exact sequences be commutative

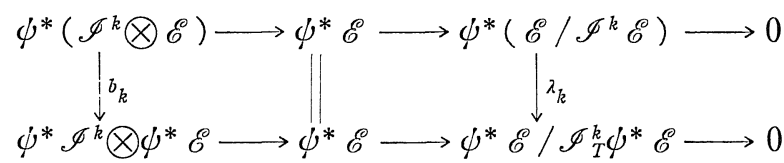

where $b_{k}$ is the inverse of the canonical isomorphism (cf. [6, 0, $(4.3 .3)]$ ) and the top (resp. the bottom) sequence is obtained by pulling back by $\phi$ the exact sequence $\mathscr{I}^{k} \otimes \mathscr{E}^{\mathscr{C}} \rightarrow \mathscr{E} \rightarrow \mathscr{E} / \mathscr{I}^{k} \mathscr{E} \rightarrow 0$ (resp. by tensoring the sequence $\phi^{*} \mathscr{I}^{k} \rightarrow \mathcal{O}_{X_{T}} \rightarrow \mathcal{O}_{X_{T}} / \mathscr{I}_{T}^{k} \rightarrow 0$ with $\left.\phi^{*} \mathscr{E}\right)$. The isomorphy of $\lambda_{k}$ is then clear.

Next, we define $\mu_{k}$ by the requirement that the following diagram of exact sequences be commutative

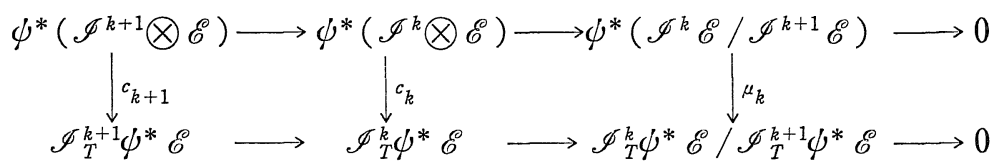

where $c_{k}$ is the composite of $b_{k}$ above and the natural surjection $\psi^{*} \mathscr{I}^{k} \otimes \psi^{*} \mathscr{E} \rightarrow \mathscr{I}_{T}^{k} \psi^{*} \mathscr{E}$ and where the top sequence is obtained from the exact sequence $\mathscr{I}^{k+1} \rightarrow \mathscr{I}^{k} \rightarrow \mathscr{I}^{k} / \mathscr{I}^{k+1} \rightarrow 0$ by applying to it $\psi^{*}(\otimes \mathscr{E})$, taking the natural isomorphism $\mathscr{I}^{k} \mathscr{E} / \mathscr{I}^{k+1} \mathscr{E} \cong \mathscr{I}^{k} / \mathscr{I}^{k+1} \otimes \mathscr{E}$ into account. The surjectivity of $\mu_{k}$ is clear. q. e.d.

2.2. Now we come to the reformulation of [3, Lemma 5.8] mentioned in the introduction. We say that a meromorphic map $g: Z \rightarrow Y$ is generically surjective if the image of $Z$ contains a dense open subset of $Y$. Moreover we employ the following notations through the end of this paper.

Notation. Let $f: X \rightarrow S$ be a morphism of complex spaces and $\mathscr{E}$ a coherent analytic sheaf on $X$. We denote by $u_{X / S}(\mathscr{E}): \mathscr{E}_{D} \rightarrow \mathscr{R}_{X / S}(\mathscr{E})$ a fixed universal quotient (homomorphism) on $X_{D}:=X \times{ }_{S} D$ (defined up to automorphisms of $\left.\mathscr{R}_{X / S}(\mathscr{E})\right)$, where $D=D_{X / S}(\mathscr{E})$. Let $Z_{X / S}$ 
$\subseteq X_{D}:=X \times{ }_{s} D$ be the universal subspace where $D=D_{X / s}$. Then as in [3] for any irreducible component $D_{\alpha}$ of $D_{X / S}(\mathscr{E})_{\text {red }}$ we shall denote $X_{D_{\alpha}}:=X \times{ }_{S} D_{\alpha}, \quad \mathscr{E}_{D_{\alpha}},\left(\mathscr{R}_{X / S}(\mathscr{E})\right)_{D_{\alpha}}$ simply by $X_{\alpha}, \mathscr{E}_{\alpha}, \mathscr{R}_{\alpha}$ respectively, and further, when $\mathscr{E}=\mathcal{O}_{X}$, denote $Z_{X / S} \times{ }_{s} D_{\alpha}$ by $Z_{\alpha}$. By $\hat{D}_{X / S}$ we denote the union of those irreducible components $D_{\alpha}$ of $D_{X / S \text {, red }}$ for which $Z_{\alpha}$ is reduced. We set $\operatorname{dim} X / S=\operatorname{dim} X-\operatorname{dim} S$ and $\operatorname{dim} \mathscr{E} / S$ $=\operatorname{dim} \operatorname{supp}(\mathscr{E})-\operatorname{dim} S$, where supp denotes the support.

Lemma 12. Let $f: X \rightarrow S$ be a proper morphism of complex spaces and $\mathscr{E}$ a coherent analytic sheaf on $X$. Then for every irreducible component $D_{\alpha}$ of $D_{X / S}(\mathscr{E})_{\text {red }}$ with $\operatorname{dim} \mathscr{R}_{\alpha} / D_{\alpha}=q \geqq 0$, there exist 1) an irreducible component $T$ of $\hat{D}_{X / S}$ such that $\operatorname{dim} Z_{T} / T=q$ where $\left.Z=Z_{X / s}, 2\right)$ coherent analytic sheaves $\mathscr{E}_{k}, 0 \leqq k \leqq n$, on $Z_{T}, 3$ ) an irreducible component $B_{k}$ of $D_{Z_{T} / T}\left(\mathscr{E}_{k}\right)_{\text {red }}$ for each $k$ such that $Z_{T} \times_{T} B_{k}$ is reduced, 4) a complex variety $A$ which is locally projective over $B_{0} \times_{T} \cdots \times_{T} B_{n}$ and finally 5) a generically surjective meromorphic $S$-map $h: A \rightarrow D_{\alpha}$.

Proof. Let $\mathscr{J}$ be the ideal sheaf of annihilators of $\mathscr{R}_{\alpha}$ on $X_{\alpha}$. Let supp $\mathscr{R}_{\alpha}$ be the support of $\mathscr{R}_{\alpha}$. Then define the subspace $S\left(\mathscr{R}_{\alpha}\right)$ of $X_{\alpha}$ by $S\left(\mathscr{R}_{\alpha}\right)=\left(\operatorname{supp} \mathscr{R}_{\alpha}, \mathcal{O} / \mathscr{J}\right)$. Let $\mathscr{I}$ be the ideal sheaf of supp $\mathscr{R}_{\alpha}$ on $X_{\alpha}$. Define $\mathscr{R}_{k}=\mathscr{I}^{k} \mathscr{R}_{\alpha} / \mathscr{I}^{k+1} \mathscr{R}_{\alpha}, k \geqq 0$, where $\mathscr{I}^{0}=\mathcal{O} \equiv$ $\mathcal{O}_{X_{\alpha}}$. Then we have the following commutative diagram of exact sequences on $X_{\alpha}$

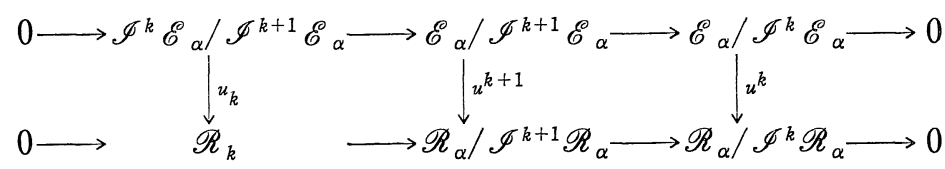

with the vertical arrows surjective. Take $n>0$ sufficiently large so that $\mathscr{J} \supseteqq \mathscr{I}^{n+1}$ over $U_{0}$ for some Zariski open subset $U_{0}$ of $D_{\alpha}$. Let $U$ be a Zariski open subset of $D_{\alpha}$ such that $U \subseteq U_{0}$ and that $\mathcal{O} / \mathscr{I}$ and $\mathscr{R}_{k}, 0 \leqq k \leqq n$, are all flat over $U$. Then by $(19)_{k}$ we see that $\mathscr{R}_{\alpha} /$ $\mathscr{I}^{k} \mathscr{R}_{\alpha}$ also are flat over $U$ for $1 \leqq k \leqq n+1$. Now since $\mathcal{O} / \mathscr{I}$ is flat over $U$, by the universality of $D_{X / S}$ we have a unique $S$-morphism $\psi: U \rightarrow D_{X / S}$ such that

$$
\left(\operatorname{supp} \mathscr{R}_{\alpha}\right)_{U} \cong Z_{U}:=Z \times_{D_{X / S}} U .
$$

Let $T$ be any irreducible component of $D_{X / S \text {, red }}$ containing $\psi(U)$. 
Then by (20) and Lemma $10 Z_{T}$ is reduced, so $T \subseteq \hat{D}_{X / S}$.

Let $\mathscr{I}_{T}$ be the ideal sheaf of $Z_{T}$ in $X_{T}$ and $\mathscr{E}_{k}=\mathscr{I}_{T}^{k} \mathscr{E}_{T} / \mathscr{I}_{T}^{k+1} \mathscr{E}_{T}$, regarded as a coherent analytic sheaf on $Z_{T}$. We then consider the relative Douady space $D_{k}:=D_{Z_{T} / T}\left(\mathscr{E}_{k}\right)$ associated with the pair $\left(Z_{T} / T\right.$, $\left.\mathscr{E}_{k}\right)$. Then by (20), Lemma 11 and $(19)_{k}, \mathscr{R}_{k}$, restricted over $U$, are flat quotients of $\left(\mathscr{E}_{k}\right)_{U} \equiv \tilde{\psi}^{*} \mathscr{E}_{k}$, where $\tilde{\psi}: Z_{U} \cong Z_{T} \times{ }_{T} U \rightarrow Z_{T}$ is the natural morphism. Then by the universality of $D_{k}, \phi$ lifts to a unique morphism $\tau_{k}: U \rightarrow D_{k}$ such that $\hat{u}_{k}:\left(\mathscr{E}_{k}\right)_{U} \rightarrow \mathscr{R}_{k}$ is isomorphic to the pullback via $\tau_{k}$ of the universal quotient $u_{Z_{T} / T}\left(\mathscr{E}_{k}\right): \mathscr{E}_{k, D_{k}} \rightarrow \mathscr{R}_{Z_{T} / T}\left(\mathscr{E}_{k}\right)$. Let $B_{k}$ be any irreducible component of $D_{k, \text { red }}$ containing $\tau_{k}(U)$. Then again by Lemma $10 Z_{T} \times_{T} B_{k}$ is reduced. Let $v_{k}: \mathscr{E}_{k, B_{k}} \rightarrow \mathscr{R}$ be the restriction of $u_{Z_{T} / T}\left(\mathscr{E}_{k}\right)$ over $B_{k}$. Define $\tau=\tau_{0} \times_{T} \cdots \times_{T} \tau_{n}: U \rightarrow$ $B:=B_{0} \times_{T} \cdots \times{ }_{T} B_{n}$. Let $\pi_{k}: B_{k} \rightarrow T, \pi: B \rightarrow T, p_{k}: B \rightarrow B_{k}$ be the natural projections, and $\tilde{\pi}_{k}: Z_{B_{k}} \rightarrow Z_{T}, \tilde{\pi}: Z_{B} \rightarrow Z_{T}, \tilde{p}_{k}: Z_{B} \rightarrow Z_{B_{k}}$ the induced morphisms.

We shall now apply Lemma 9 to our situation;

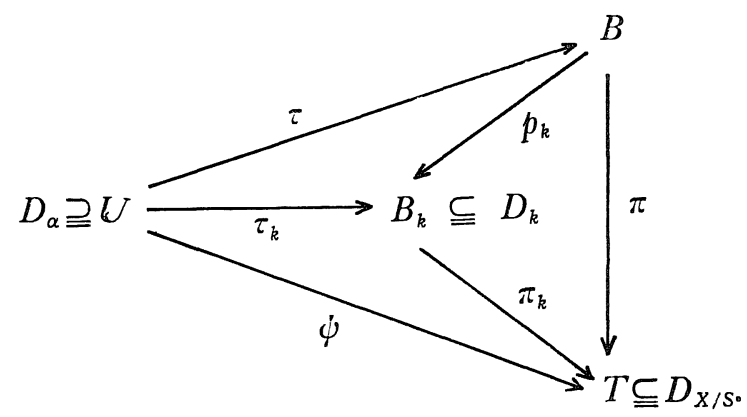

In the notation of 1.4 first we set $m=n$ and

$$
\left\{\begin{array}{l}
(f: X \rightarrow B)=\left(f_{B}: X_{B} \rightarrow B\right) \\
\left(\mathscr{E}_{k}, \mathscr{E}^{k}\right)=\left(\tilde{\pi}^{*} \mathscr{E}_{k}, \tilde{\pi}^{*}\left(\mathscr{E}_{T} / \mathscr{I}_{T}^{k+1} \mathscr{E}_{T}\right)\right) \\
\left(u_{k}: \mathscr{E}_{k} \rightarrow \mathscr{R}_{k}\right)=\left(\tilde{p}_{k}^{*}\left(v_{k}\right): \tilde{\pi}^{*} \mathscr{E}_{k}=\tilde{p}_{k}^{*}\left(\tilde{\pi}_{k}^{*} \mathscr{E}_{k}\right) \rightarrow \tilde{p}_{k}^{*}\left({ }_{k} \mathscr{R}\right)\right)^{1)} .
\end{array}\right.
$$

Then $\mathscr{E}^{0}=\mathscr{E}_{0}$ and we have a natural exact sequence $\mathscr{E}_{k} \rightarrow \mathscr{E}^{k} \rightarrow \mathscr{E}^{k-1}$ $\rightarrow 0$. We further set

$$
\left\{\begin{array}{l}
(\tau: U \rightarrow B)=(\tau: U \rightarrow B) \\
\left(u^{k}: \mathscr{E}_{U}^{b} \rightarrow \mathscr{R}^{k}\right)=\left(u_{U}^{k+1}:\left(\mathscr{E}_{\alpha} / \mathscr{I}^{k+1} \mathscr{E}_{\alpha}\right)_{U} \rightarrow\left(\mathscr{R}_{\alpha} / \mathscr{I}^{k+1} \mathscr{R}_{\alpha}\right)_{U}\right)
\end{array}\right.
$$

where we identified $\tilde{\tau}^{*}\left(\tilde{\pi}^{*}\left(\mathscr{E}_{T} / \mathscr{I}_{T}^{k+1} \mathscr{E}_{T}\right)\right) \cong \tilde{\psi}^{*}\left(\mathscr{E}_{T} / \mathscr{I}_{T}^{k+1} \mathscr{E}_{T}\right)$ with

1) We consider $\mathscr{E}_{k}, \mathscr{E}^{k}, \mathscr{R}_{k}$ as coherent analytic sheaves on $X_{B}$ with respect to the natural inclusion $Z_{B}:=Z_{T} \wedge{ }_{T} B \subseteq X_{B}$, where these sheaves are zero outside $Z_{B}$. 
$\left(\mathscr{E}_{\alpha} / \mathscr{I}^{k+1} \mathscr{E}_{\alpha}\right)_{U} \cong \tilde{\psi}^{*} \mathscr{E}_{T} / \mathscr{I}^{k+1} \tilde{\phi}^{*} \mathscr{E}_{T}$ with respect to the natural isomorphism $\tilde{\phi}^{*}\left(\mathscr{E}_{T} / \mathscr{I}^{k+1} \mathscr{E}_{T}\right) \cong \tilde{\psi}^{*} \mathscr{E}_{T} / \mathscr{I}^{k+1} \tilde{\psi}^{*} \mathscr{E}_{T}$ given in Lemma $9^{2}$. With this definition $u^{0}=u_{0, T}$ by the definition of $\tau_{0}$ and $u_{0}$, and we get the commutative diagram $(16)_{k}$ on $X_{U}$ in view of $(19)_{k}$.

Thus we can apply Lemma 9 to these data; we can find a complex variety $A$ which is locally projective over $B$, a dense open subset $V \cong U$, a $B$-morphism $\eta: V \rightarrow A$ which lifts $\left.\tau\right|_{V}$, and a coherent quotient $u(A): \mathscr{E}_{A}^{n} \cong\left(\mathscr{E}_{B}^{n}\right)_{A} \rightarrow \mathscr{R}(A)$ on $X_{A}=X \times{ }_{S} A$, where $\mathscr{E}^{n}$ is defined by (21) such that a) $u^{n+1}: \mathscr{E}_{\alpha} / \mathscr{I}^{n+1} \mathscr{E}_{\alpha} \rightarrow \mathscr{R}_{\alpha} / \mathscr{I}^{n+1} \mathscr{R}_{\alpha}$ restricted to $X_{V}$ is isomorphic to the pull-back of $u(A)$ via $\eta$ and b) $A$ is the analytic closure of $\eta(V)$ in $A$. Let $W \subseteq A$ be a Zariski open subset over which $\mathscr{R}(A)$ is flat. Then by the universality of $D_{X / S}(\mathscr{E})$ there exists a unique morphism $h_{0}: W \rightarrow D_{X / S}(\mathscr{E})$ over $S$ such that the composite quotient $\mathscr{E}_{A} \rightarrow \mathscr{E}_{A}^{n} \rightarrow \mathscr{R}(A)$ restricted to $X_{W}$ is isomorphic to the pull-back of the universal quotient $u_{X / S}(\mathscr{E}): \mathscr{E}_{D} \rightarrow \mathscr{R}_{X / S}(\mathscr{E})$ by $h_{0}$ where $D=D_{X / S}(\mathscr{E})$. Then by the condition b) above $W_{0}:=\eta^{-1}(W)$ is a nonempty Zariski open subset of $V$ and by the condition a) $h_{0}\left(\left.\eta\right|_{W_{0}}\right)$ is the identity of $W_{0}$. (Note that $\mathscr{E}_{\alpha} / \mathscr{I}^{n+1} \mathscr{E}_{\alpha}=\mathscr{E}_{\alpha}$ on $X_{V}$ ) Hence $W_{0} \subseteq h_{0}(W)$, and then since $W$ is irreducible, $h_{0}(W) \leqq D_{\alpha}$. Finally by [4, Lemma 4] $h_{0}$ extends to a meromorphic map $h: A \rightarrow$ $D_{\alpha}$ which is generically surjective. This completes the construction of all the objects required in the lemma.

q. e. d.

2.3. For the proof of Theorem, besides Lemma 12 above we need Lemma 5.9 and Lemma 5.7 of [3], which we shall quote here as Lemma 13 and Lemma 14 respectively.

Lemma 13. Let $f: X \rightarrow S$ be a proper flat morphism of reduced complex spaces and $\mathscr{E}$ a coherent analytic sheaf on $X$. Let $q=\operatorname{dim} X / S$. Then for any irreducible component $D_{\alpha}$ of $D_{X / S}(\mathscr{E})_{\mathrm{red}}$ such that $X_{\alpha}$ is reduced, there exist 1) irreducible components $T_{i}, 1 \leqq i \leqq m$, of $\hat{D}_{X / s}$ such that $Z_{i}:=Z_{X / S} \times{ }_{S} T_{i}$ are irreducible, 2) for each $i$ subvariety $Y_{i}$ of $D_{X_{i} / T_{i}}\left(\mathscr{E}_{i}\right)_{\text {red }}$, where $X_{i}=X_{T_{i}}$ and $\mathscr{E}_{i}=\mathscr{E}_{T_{i}}$, such that either a) $\operatorname{dim} \mathscr{R}_{i} / Y_{i}$ $<q$ or b) $\tilde{Z}_{i}:=Z_{i} \times_{T_{i}} Y_{i}$ is reduced, $\mathscr{R}_{i}$ is a torsion free $\mathcal{O}_{\tilde{Z}_{2}}$-module and $Y_{i} \subseteq D_{Z_{\imath} / T_{i}}\left(\overline{\mathscr{E}}_{i}\right)$ with respect to the natural inclusion $D_{Z_{\iota} / T_{i}}\left(\overline{\mathscr{E}}_{i}\right) \leqq D_{X_{i} / T_{i}}\left(\mathscr{E}_{i}\right)$

2) $\tilde{\tau}$ is the natural morphism $Z_{T} \wedge_{T} U \rightarrow Z_{T} \wedge_{T} B$ induced by $\tau$. 
where $\mathscr{R}_{i}=\left(\mathscr{R}_{X_{i} / T_{i}}\left(\mathscr{E}_{i}\right)\right)_{Y_{i}}$ and $\left.\overline{\mathscr{E}}_{i}=\mathscr{E}_{i} \otimes_{\mathcal{O}_{X_{i}}} \mathcal{O}_{Z_{i}}, 3\right)$ an analytic subvariety $N$ of $Y_{1} \times_{s} \ldots \times_{s} Y_{m}$, and finally 4) a generically surjective meromorphic map $h: N \rightarrow D_{\alpha}$ over $S$.

The newly added statement ' $Y_{i} \subseteq D_{Z_{i} / T_{i}}\left(\overline{\mathscr{E}}_{i}\right)^{\prime}$ above is in fact shown in the final part of the proof of Lemma 5.9 of [3].

Lemma 14. Let $f: X \rightarrow S$ be a proper flat morphism of complex varieties and $\mathscr{E}$ a coherent analytic sheaf on $X$. Let $r>0$ be an integer and $Y=X \times{ }_{s} G_{r}(\mathscr{E})$, where $G_{r}(\mathscr{E})$ is the Grassmann variety over $X$ of locally free quotients of rank $r$ cf $\mathscr{E}$ (cf. [6]), regarded naturally as a complex space over $S$. Then for any irreducible component $D_{\alpha}$ of $D_{X / S}(\mathscr{E})_{\text {red }}$ such that $X_{\alpha}$ is reduced and that $\mathscr{R}_{\alpha}$ is torsion free of rank $r$ on $X_{\alpha}$ there exist an analytic subset $E_{\alpha}$ of $\hat{D}_{Y / s}$ and a bimeromorphic map $\tau: E_{\alpha} \rightarrow D_{\alpha}$ over $S$.

Here we remark that the proof of this lemma in [3] uses [3, Lemma 5.5], but unfortunately its proof is incomplete. (Perhaps not true as is stated there.) So we shall formulate and prove another version of it (Proposition 3 below), which is enough for Lemma 14 above as follows immediately from the proof of Lemma 5.7 in [3].

Let $T$ be a complex variety and $h: Y \rightarrow T$ be a morphism of complex spaces with $Y$ reduced. Let $Y_{i}, 1 \leqq i \leqq m$, be the irreducible components of $Y$. Then we say that $Y$ is pure dimensional over $T$ if $Y$ is pure dimensional and each $Y_{i}$ is mapped surjectively onto $T$. In this case $\operatorname{dim} Y_{i} / T=\operatorname{dim} Y / T$ is independent of $i$.

To state the proposition we introduce some notations. Let $f: X \rightarrow S$, $f^{\prime}: X^{\prime} \rightarrow S$ be proper morphisms of complex spaces. Let $Z \cong X \times{ }_{S} X^{\prime}$ be a subspace. Then we set

$$
\begin{aligned}
M_{0}= & \left\{s \in S ; X_{s} \text { is reduced and } Z_{s} \subseteq X_{s} \times X_{s}^{\prime}\right. \text { is a graph of a } \\
& \text { meromorphic map } \left.X_{s} \rightarrow X_{s}^{\prime}\right\} .
\end{aligned}
$$

Assume further that there exists an $S$-morphism $g: X^{\prime} \rightarrow X$. Then we further set

$M=\left\{s \in S ; X_{s}\right.$ is reduced and $Z_{s}$ is a graph of a meromorphic section of $\left.g_{s}: X_{s}^{\prime} \rightarrow X_{s}\right\}$. 
Proposition 3. Let $f: X \rightarrow S, f^{\prime}: X^{\prime} \rightarrow S, Z \subseteq X \times{ }_{S} X^{\prime}$ and $g: X^{\prime} \rightarrow X$ be as above. Suppose that both $X$ and $Z$ are reduced and $S$ is a variety. Suppose further that both $X$ and $Z$ are pure dimensional over $S$. Then

1) $Z$ is a graph of a meromorphic map $X \rightarrow X^{\prime}$ over $S$ if and only if there exists a Zariski open subset $U \subseteq S$ such that for any $s \in U, X_{s}$ is reduced and $Z_{s}$ is a graph of a meromorphic map $X_{s} \rightarrow X_{s}^{\prime}$.

2) Let $h: Z \rightarrow S$ be the natural morphism. Suppose that both $f$ and $h$ are flat. Then $M_{0}$ is Zariski open in $S$ (possibly empty) and $M$ is locally closed with respect to the Zariski topology of $S$.

First we make the following obvious remark. Let $g: \tilde{Y} \rightarrow Y$ be a morphism of complex spaces. Then

$$
\left\{\begin{array}{l}
g \text { is isomorphic if and only if } 1) \operatorname{dim}_{\tilde{y}} g^{-1} g(\tilde{y})=0 \\
\text { for any } \tilde{y} \in \tilde{Y} \text { and 2) the natural homomorphism } \\
\iota_{g}: \mathcal{O}_{Y} \rightarrow g_{*} \mathcal{O}_{\tilde{Y}} \text { is isomorphic. }
\end{array}\right.
$$

The essential part of the proof of the proposition is contained the following

Lemma 15. Let $S$ be a complex variety. Let $f: X \rightarrow S, h: Z \rightarrow S$ be morphisms of reduced complex spaces and $\pi: Z \rightarrow X$ an $S$-morphism. Suppose that $X$ and $Z$ are pure dimensional over $S$. Let $U_{f}:=\{s \in S ; f$ is flat along $X_{s}$ and $X_{s}$ is reduced $\}$ and $U_{h}:=\left\{s \in S ; h\right.$ is flat along $Z_{s}$ and $Z_{s}$ is reduced $\}$. Then the set $N:=\left\{s \in U_{f} \cap U_{h} ; \pi_{s}: Z_{s} \rightarrow X_{s}\right.$ is bimeromorphic $\}$ is Zariski open in $S$. Moreover $N$ is nonempty if and only if $\pi$ is bimeromorphic.

Proof. First we note that $U_{f}$ and $U_{h}$ are Zariski open by [3, Lemma 1.5]. We set $U_{0}=U_{f} \cap U_{h}$. Let $p=\operatorname{dim} Z / S$ and $q=\operatorname{dim} X / S$. Then

(23) $Z_{s}$ (resp. $X_{s}$ ) has pure dimension $p$ (resp. $q$ ) for any $s \in U_{0}$. Let $\tilde{A}=\left\{z \in Z ; \operatorname{dim}_{z} \pi^{-1} \pi(z)>0\right\}$ and $A=\pi(\tilde{A})$. Then clearly $\tilde{A}_{s}=\{z$ $\left.\in Z_{s} ; \operatorname{dim}_{z} \pi_{s}^{-1} \pi_{s}(z)>0\right\}$. Consider now the exact sequence

$$
0 \longrightarrow \mathscr{K} \longrightarrow \mathcal{O}_{X} \stackrel{\iota \pi}{\longrightarrow} \pi_{*} \mathcal{O}_{Z} \longrightarrow \mathscr{M} \longrightarrow 0
$$

of coherent analytic sheaves on $X$ where $\mathscr{K}$ (resp. $\mathscr{M}$ ) is the kernel (resp. image) of $\iota_{\pi}$. Let $B=\operatorname{supp} \mathscr{M}$ and $C=\operatorname{supp} \mathscr{K}$. Let $W_{1}=$ $X-A, W_{2}=X-(A \cup B)$ and $W_{3}=X-(A \cup B \cup C)$. Let $\tilde{W}_{i}=\pi^{-1}\left(W_{i}\right)$ 
and $\pi_{i}=\left.\pi\right|_{\tilde{W}_{i}}: \tilde{W}_{i} \rightarrow W_{i}$. Then $\pi_{1}: \tilde{W}_{1} \rightarrow W_{1}$ is a finite morphism, and $\pi_{3}: \tilde{W}_{3} \rightarrow W_{3}$ is an isomorphism in view of (22). In fact, $W_{3}$ is the maximal Zariski open subset of $X$ with this property. Further

$$
B_{s}=\operatorname{supp} \pi_{s *} \mathcal{O}_{z_{s}} / \iota_{\pi_{s}} \mathcal{O}_{X_{s}} \text { on } W_{1} \cap X_{s} \text {. }
$$

This can be seen as follows. By the restriction to each fiber $X_{s}$ (24) gives the exact sequence

$$
\mathcal{O}_{X_{s}} \stackrel{\left(\iota_{\pi}\right)_{s}}{\longrightarrow}\left(\pi_{*} \mathcal{O}_{2}\right)_{s} \longrightarrow \mathscr{M}_{s} \longrightarrow 0 .
$$

On the other hand, since $\pi_{1}$ is finite, on $X_{s} \cap W_{1}$ there exists a natural isomorphism between $\left(\ell_{\pi}\right)_{s}: \mathcal{O}_{X_{s}} \rightarrow\left(\pi_{*} \mathcal{O}_{Z}\right)_{s}$ and $\iota_{\pi_{s}}: \mathcal{O}_{X_{s}} \rightarrow \pi_{s *} \mathcal{O}_{z_{s}}$ Thus supp $\pi_{s *} \mathcal{O}_{z_{s}} / \iota_{\pi_{s}} \mathcal{O}_{X_{s}}=\operatorname{supp} \mathscr{M}_{s}$ and (25) follows. Next we shall see that

$$
C_{s}=\operatorname{supp}\left(\operatorname{Ker} \iota_{\pi_{s}}\right) \text { on } W_{2} \cap f^{-1}\left(U_{h}\right) .
$$

Indeed, on $W_{2}$ (24) reduces to $0 \rightarrow \mathscr{K} \rightarrow \mathcal{O}_{X} \rightarrow \pi_{*} \mathcal{O}_{Z} \rightarrow 0$ and on $W_{1} \cap$ $f^{-1}\left(U_{h}\right), \pi_{*} \mathcal{O}_{Z}$ is $f$-flat. Thus for any $s \in U_{h}$ the restriction of (24) to $X_{s}$ gives the exact sequence

$$
0 \longrightarrow \mathscr{K}_{s} \longrightarrow \mathcal{O}_{X_{s}} \stackrel{\iota \pi_{s}}{\longrightarrow} \pi_{s *} \mathcal{O}_{z_{s}} \longrightarrow 0
$$

on $W_{2} \cap X_{s}$. Thus supp $\left(\operatorname{Ker}{\iota_{\pi}}_{s}\right)=\operatorname{supp} \mathscr{K}_{s}$ and (26) follows.

Now set $T_{A}=\left\{s \in S ; \operatorname{dim} \tilde{A}_{s} \geqq p\right\}, T_{B}=\left\{s \in S ; \operatorname{dim} B_{s} \geqq q\right\}$, and $T_{C}=\left\{s \in S ; \operatorname{dim} C_{s} \geqq q\right\}$. Let $U_{1}=S-\left(T_{A} \cup T_{B} \cup T_{C}\right)$. Then the first assertion follows from the following:

Claim. $N=U_{0} \cap U_{1}$.

Proof. It is clear that $N \leqq U_{0}$. Let $s \in N$ be arbitrary. Then $\pi_{s}: Z_{s} \rightarrow X_{\mathrm{s}}$ is bimeromorphic. In particular $s \notin T_{A}$ by (23) and hence $W_{1} \cap X_{s}$ is dense in $X_{s}$. It follows then that $s \notin T_{B}$ by (25) and so $W_{2} \cap X_{s}$ is also dense in $X_{s}$. Then by (26) $s \notin T_{C}$ either. Thus $s \in$ $U_{1}$ and we have proved that $N \leqq U_{0} \cap U_{1}$. Conversely, let $s \in U_{0} \cap U_{1}$ be an arbitrary point. Then by (23) $\tilde{A}_{s}$ is nowhere dense in $Z_{s}$ and then $\pi_{s}$ is generically finite. Thus $\pi_{s}\left(\tilde{A}_{s}\right)=A_{s}$ is nowhere dense in $X_{s}$. Hence $X_{s} \cap W_{3}$ is dense in $X_{s}$ and $Z_{s} \cap \tilde{W}_{3}$ in $Z_{s}$. Thus $\pi_{s}$ is bimeromorphic and $s \in N$.

By the above claim it follows that $N \neq \emptyset$ if and only if $U_{1} \neq \emptyset$. Further by the pure dimensionality assumption the latter condition 
is equivalent to the condition that $W_{3}$ is dense in $X$ and $\tilde{W}_{3}$ is dense in $Z$. From this the desired equivalence follows. q. e.d.

Proof of Proposition 3. Let $\pi: Z \rightarrow X$ be the natural morphism. Let $N$ be defined as in Lemma 15 applied to $\pi$. 1) If $Z$ is a graph of a meromorphic map, $\pi$ is bimeromorpnic. Then it suffices to set $U=N$. Conversely suppose that a Zariski open subset $U$ satisfying the condition of 1 ) exists. Restricting $U$, we may assume that $f, h$ are flat over $U$ and $Z_{s}$ is reduced for any $s \in S$ (cf. [3]). Since $Z_{s}$ is a graph of a meromorphic map if and only if $\pi_{s}: Z_{s} \rightarrow X_{s}$ is bimeromorphic, $U$ is contained in $N$. Hence $N \neq \emptyset$ and then $\pi$ is bimeromorphic by Lemma 15.

2) Since both $f$ and $h$ are flat, $N=\left\{s \in S ; X_{s}, Z_{s}\right.$ are reduced, and $\pi_{s}: Z_{s} \rightarrow X_{s}$ is bimeromorphic\}. Then clearly $N \leqq M_{0}$. Conversely, if $s \in M_{0}$, then $Z_{s}$ is reduced since $X_{s}$ is reduced. Thus $s \in N$. Hence $N=M_{0}$ and $M_{0}$ is Zariski open. Once this is established, the proof for $M$ is the same as that of Lemma 5.5 2) in [3].

2.4. Using Lemmas 12,13 , and 14 above and Theorem in [4] (cf. Introduction) we shall prove Theorem along the line of Proof of Theorem 5.2 in [3].

Proof of Theorem. First we assume that $f$ is a $\mathscr{C}$-morphism. For the functorial properties of $\mathscr{C}$-morphisms used below we refer to [4. (2.4)]. The statement of Theorem is clearly equivalent to the following :

Let $D_{\alpha}$ be any irreducible component of $D_{X / S}(\mathscr{E})_{\text {red. }}$. Then for any irreducible component $D_{\alpha, Q}^{i}$ of $D_{\alpha, Q}:=D_{\alpha} \times{ }_{s} Q$, the natural morphism $b_{\alpha, Q}^{i}: D_{\alpha, Q}^{i} \rightarrow Q$ (is proper and) is a $\mathscr{C}$-morphism.

We prove Theorem in this form by induction on $q=\operatorname{dim} \mathscr{R}_{\alpha} / S$. If $q=-1$ (i. e., supp $\mathscr{R}_{\alpha}=\emptyset$ ), then $\mathscr{R}_{\alpha}=\{0\}$ so that $D_{\alpha} \cong S$ and hence the theorem is clearly true. So assume that $q \geqq 0$ in what follows.

Step 1. We may assume that $f$ is flat, $\operatorname{dim} X / S=q$, and that both $X$ and $X_{\alpha}$ are reduced.

Proof. Suppose that the theorem is true in this case. We look 
at Lemma 12 applied to $f, \mathscr{E}$ and $D_{\alpha}$. We use the notations of that lemma. First of all, since $T$ is an irreducible component of $\hat{D}_{X / S}$ and $\rho_{T}: Z_{T} \rightarrow T$ is a $\mathscr{C}$-morphism (being induced by the projection $f_{T}: X_{T} \rightarrow T$ ), for any irreducible component $T_{Q}^{i}$ of $T_{Q}$ the natural morphism $T_{Q}^{i} \rightarrow Q$ is a $\mathscr{C}$-morphism by Theorem in [4]. Moreover, we note that $T_{Q}^{i}$ is actually relatively compact in $T$ as we see immediately by applying the above argument to any relatively compact $Q^{\prime} \subseteq S$ with $Q \Subset Q^{\prime}$. (The same kind of remark applies also to the other spaces defined below, though we do not mention it explicitly.) On the other hand, $\rho_{T}$ is flat, $\operatorname{dim} Z_{T} / T=q$ and for any $0 \leqq k \leqq n \quad Z_{T} \times{ }_{T} B_{k}$ is reduced. Thus Theorem is true for $\left(\rho_{T}: Z_{T} \rightarrow T\right.$, $\mathscr{E}_{k}, B_{k}$ ) for each $k$ by our assumption. Hence by the above remark for any irreducible component $B_{k}^{i}:=B_{k, Q}^{i}$ of $B_{k, Q}$ the natural morphism $B_{k}^{i} \rightarrow T_{Q}$ is a $\mathscr{C}$-morphism. Hence for any $I=\left(i_{0}, \ldots, i_{m}\right) B_{0}^{i_{0}} \times_{T_{Q}} \cdots$ $\times_{T_{Q}} B_{n}^{i_{n}} \rightarrow T_{Q}$ is a $\mathscr{C}$-morphism, and so the composite map

$$
A_{Q}^{I} \longrightarrow B_{0}^{i_{0}} \times_{T_{Q}} \cdots \times_{T_{Q}} B_{n}^{i_{n}} \longrightarrow T_{Q} \longrightarrow Q
$$

also is a $\mathscr{C}$-morphism, where $A_{Q}^{I}$ is the inverse image of $B_{0}^{i_{0}} \times{ }_{T_{Q}} \cdots$ $\times_{T_{Q}} B_{n}^{i n}$ in $A$. From this it follows that $b_{\alpha, Q}^{i}$ is a $\mathscr{C}$-morphism by Lemma 12.

Step. 2. In addition to the conditions of Step 1 we may further assume that both $X$ and $S$ are irreducible and that $\mathscr{R}_{\alpha}$ is torsion free on $X_{\alpha}$.

Proof. Suppose that Theorem is proved under this assumption. We observe Lemma 13 applied to the given $f, \mathscr{E}$ and $D_{\alpha}$ and use the notations of that lemma. First since $T_{i} \subseteq \hat{D}_{X / S}$, for any irreducible component $T_{i}^{r}:=T_{i, Q}^{\gamma}$ of $T_{i, Q}$, the natural morphism $T_{i}^{r} \rightarrow Q$ is a $\mathscr{C}$-morphism by Theorem in [4]. Hence for any $\left(\gamma_{1}, \ldots, \gamma_{m}\right)$ the induced morphism $T_{1}^{\gamma_{1}} \times_{Q} \cdots \times_{Q} T_{m}^{\gamma_{m}} \rightarrow Q$ also is a $\mathscr{C}$-morphism. Next we shall show that for any irreducible component $Y_{i}^{\mu}:=Y_{i, Q}^{\mu}$ of $Y_{i, Q}$ the natural morphism $Y_{\imath}^{\mu} \rightarrow T_{i, Q}$ is a $\mathscr{C}$-morphism, and hence that for any $M=\left(\mu_{1}, \ldots, \mu_{m}\right)$ the induced morphism $N_{Q}^{M}:=N_{Q} \cap\left(Y_{1}^{\mu_{1}} \times_{Q}\right.$ $\left.\cdots \times \times_{Q} Y_{m}^{\mu_{m}}\right) \rightarrow T_{Q}:=T_{1, Q} \times_{Q} \cdots \times_{Q} T_{m, Q}$ also is a $\mathscr{C}$-morphism. This would then imply that the composite map $N_{Q}^{M} \rightarrow T_{Q} \rightarrow Q$ is again a 
$\mathscr{C}$-morphism. Hence by Lemma $13 b_{\alpha, Q}^{i}$ would also be a $\mathscr{C}$-morphism.

Now we show that

$$
Y_{i}^{\mu} \rightarrow T_{i, Q} \text { is a } \mathscr{C} \text {-morphism. }
$$

Suppose first that $\operatorname{dim} \mathscr{R}_{i} / Y_{i}=q$. Let $Y_{\beta_{i}}$ be any irreducible component of $D_{Z_{i} / T_{i}}\left(\overline{\mathscr{E}}_{i}\right)_{\text {red }}$ containing $Y_{i}$. Then we claim that the triple $\left(\rho_{i}: Z_{i} \rightarrow T_{i}, \overline{\mathscr{E}}_{i}, Y_{\beta_{i}}\right)$ satisfies the condition of Step 2. First, by Lemma $13 Z_{i}$ and $T_{i}$ are varieties, $\rho_{i}$ is flat and $\operatorname{dim} Z_{i} / T_{i}=\operatorname{dim} \tilde{Z}_{i} /$ $Y_{i}=\operatorname{dim} \mathscr{R}_{i} / Y_{i}=q$. Further by Lemma $10 \tilde{Z}_{\beta_{i}}:=Z_{i} \times_{T_{i}} Y_{\beta_{i}}$ is reduced since $\tilde{Z}_{i}=\left.\tilde{Z}_{\beta_{i}}\right|_{Y_{i}}$ is reduced. Similarly, $\mathscr{R}_{\beta_{i}}:=\mathscr{R}_{Z_{i} / T_{i}}\left(\overline{\mathscr{E}}_{i}\right)_{\beta_{i}}$ is a torsion free $\mathcal{O}_{\tilde{Z}_{\beta_{i}}}$-module by Lemma 10 since $\tilde{Z}_{\beta_{i}}$ is pure dimensional as well as $Z_{i}$ (cf. [4, Lemma 3]) and $\mathscr{R}_{i}=\mathscr{R}_{Z_{i} / T_{i}}\left(\overline{\mathscr{E}}_{i}\right)_{Y_{i}}$ is torsion free. Thus our claim is proved. Hence from our assumption (27) follows. Next assume that $\operatorname{dim} \mathscr{R}_{i} / Y_{i}<q$. Then $\operatorname{dim} \mathscr{R}_{\beta_{i}} / Y_{\beta_{i}}<q$ also by the flatness of $\mathscr{R}_{\beta_{i}}$ over $Y_{\beta_{i}}$. Hence by induction hypothesis (27) again follows.

Step 3. By Step 2 we may assume that $f$ and $D_{\alpha}$ satisfy the condition of Step 2. This time we observe Lemma 14 applied to our $f, \mathscr{E}$ and $D_{\alpha}$. We use the notations of that lemma. Since $E_{\alpha} \subseteq \hat{D}_{Y / S}$ and $Y \rightarrow S$ is a $\mathscr{C}$-morphism $\left(G_{r}(\mathscr{E}) \rightarrow X\right.$ being projective), by Theorem in [4] for any irreducible component $E_{\alpha, Q}^{i}$ of $E_{\alpha, Q}$ the natural morphism $E_{\alpha, Q}^{i} \rightarrow Q$ is a $\mathscr{C}$-morphism. Then the theorem follows from Lemma 14 because $D_{\alpha, Q}^{i}$ is a bimeromorphic image of some $E_{\alpha, Q}^{i}$.

In the case where $f$ is Moishezon, the above proof works without any change if we replace ' $\mathscr{C}$-morphism' by 'Moishezon morphism' there. (See (1.5) and Proposition 1 of [4] for the functorial properties instead of $[4,(2.4)])$.

q. e. d.

Remark 2. In [3, Theorem 5.2] we asserted that any irreducible component of $D_{X / S}(\mathscr{E})_{\text {red }}$ is proper over the whole $S$ without restricting to a relatively compact subdomain $Q$ as above under the assumption that $f$ is a Kähler morphism. However, this seems to be incorrect; the gap in the proof lies in the final statement of Lemma 5.7 of [3] based on the false claim that the composite map $G_{r}(\mathscr{E})$ $\rightarrow X \rightarrow S$ is again Kähler in the notation of Lemma 12, (which is in general true only after restricted over $Q$ as above (cf. [4, §2])).

At the end of [3] we also asserted that Theorem is true also for 
$f: X \rightarrow S$ in loc $-\mathscr{C} / S$ in the sense of $[4,(2.3)]$ (in $\mathscr{C} / S$ in the sense of [3]). But this is obviously false, e. g., for the fiber bundle $f: X \rightarrow$ $C$ constructed in [3, Remark 4.3], though the proof indicated there applies to a $\mathscr{C}$-morphism in the sence of [4], i. e., of this paper, as we have seen above.

\section{References}

[1] Banica, C. and Stanasila, O., Algebraic methods in the global theory of complex spaces, John Wiley \& Sons and Editura Academiei, 1976.

[2] Frisch, J., Points de platitude d'un morphisme d'espaces analytiques complexes, Inventiones math., 4 (1967), 118-138.

[3] Fujiki, A., Closedness of the Douady spaces of compact Kähler spaces, Publ. RIMS, Kyoto Univ., 14 (1978), 1-52.

[4] On the Douady space of a compact complex space in the category $C$, Nagoya Math. J., 85 (1982), 189-211.

[5] Grothendieck, A. and Dieudonné, J., Eléments de géométrie algebrique I, Publ. Math. IHES, 4 (1960).

[6] Grothendieck, A., Technique de construction en géométrie analytique, Seminaire $H$. Cartan, 13 e année, 12, 1960/61.

[7] Gunning, R.C. and Rossi, H., Analytic functions of several complex variables, Prentice Hall, Englewood Cliffs, N. J., 1965.

[8] Rossi, H., Picard varieties of an isolated singular point, Rice Univ. Studies, 54 (1968), 63-73. 
